\title{
PRPF6 promotes androgen receptor/androgen receptor- variant 7 actions in castration-resistant prostate cancer cells
}

Wei Liu ${ }^{1}$, Chunyu Wang ${ }^{1}$, Shengli Wang ${ }^{1}$, Kai Zeng ${ }^{1}$, Shan Wei ${ }^{1}$, Ning Sun ${ }^{1}$, Ge Sun ${ }^{1}$, Manlin Wang ${ }^{1}$, Renlong Zou $^{1}$, Wensu Liu ${ }^{1}$, Lin Lin ${ }^{1}$, Huijuan Song ${ }^{1}$, Zining Jin ${ }^{1,3}$, and Yue Zhao ${ }^{1,2} \llbracket$

1. Department of Cell Biology, Key laboratory of Cell Biology, Ministry of Public Health, and Key laboratory of Medical Cell Biology, Ministry of Education, School of Life Sciences, China Medical University, Shenyang City, Liaoning Province110122, China.

2. Department of Molecular Oncology, Liao Ning Tumor Hospital, Shenyang, Liaoning 110042, China.

3. Department of Breast Surgery, the First Affiliated Hospital of China Medical University, Shenyang City 110001, Liaoning Province, China.

$\triangle$ Corresponding author: Yue Zhao, E-mail: yzhao30@cmu.edu.cn; Department of Cell Biology, Key laboratory of Cell Biology, Ministry of Public Health, and Key laboratory of Medical Cell Biology, Ministry of Education, School of Life Sciences, China Medical University, No.77 Puhe Road, Shenyang North New Area, Shenyang City, Liaoning Province, China, 110122. Tel: +86 24 31939077; Fax: +86 2431939077.

(C) The author(s). This is an open access article distributed under the terms of the Creative Commons Attribution License (https://creativecommons.org/licenses/by/4.0/). See http:/ /ivyspring.com/terms for full terms and conditions.

Received: 2020.07.18; Accepted: 2020.10.20; Published: 2021.01.01

\begin{abstract}
Androgen receptor (AR) and its variants play vital roles in development and progression of prostate cancer. To clarify the mechanisms involved in the enhancement of their actions would be crucial for understanding the process in prostate cancer and castration-resistant prostate cancer transformation. Here, we provided the evidence to show that pre-mRNA processing factor 6 (PRPF6) acts as a key regulator for action of both AR full length (AR-FL) and $A R$ variant 7 (AR-V7), thereby participating in the enhancement of $A R-F L$ and AR-V7-induced transactivation in prostate cancer. In addition, PRPF6 is recruited to cis-regulatory elements in AR target genes and associates with JMJDIA to enhance AR-induced transactivation. PRPF6 also promotes expression of AR-FL and AR-V7. Moreover, PRPF6 depletion reduces tumor growth in prostate cancer-derived cell lines and results in significant suppression of xenograft tumors even under castration condition in mouse model. Furthermore, PRPF6 is obviously highly expressed in human prostate cancer samples. Collectively, our results suggest PRPF6 is involved in enhancement of oncogenic AR signaling, which support a previously unknown role of PRPF6 during progression of prostate cancer and castration-resistant prostate cancers.
\end{abstract}

Key words: androgen receptor; pre-mRNA processing factor 6; alternative splicing; transcriptional regulation; prostate cancer

\section{Introduction}

Prostate cancer is one of the leading causes of male cancer-related deaths worldwide [1]. The growth of prostate cancer is controlled by androgen/ androgen receptor (AR) axis. Androgen depletion therapy is the primary treatment for advanced prostate cancer and initially effective for tumor suppression [2]. However, a majority of patients eventually progress to a hormone resistant or castration-resistant prostate cancer (CRPC) within 2-3 years. Thus, improved therapeutic target for CRPC remains an urgent requirement.

$\mathrm{AR}$ acting as a hormone inducible transcription factor is a member of the nuclear receptor superfamily. Full-length AR (AR-FL) mainly consists of ligand-independent activation function (AF-1) and ligand-dependent activation function (AF-2). By alternative splicing process, C-terminal truncated variants of AR (AR-Vs) are generated from AR gene. AR-Vs are constitutively active and predominantly distributed in the nucleus, allowing them to drive gene transcription independent of ligand binding $[3$, 4]. AR-FL and AR-Vs along with the downstream signals are considered as the key drivers for CRPC progression $[5,6]$. Accumulating evidences demonstrate that CRPC remains dependent on persistent AR signaling, which is driven by increased androgen synthesis, enhanced nuclear transport of $\mathrm{AR}$, activated $A R$ enhancer, $A R$ amplification, 
overexpression of AR co-regulators, and generation of AR-Vs [5, 7-10]. It has been considered that aberrant expression of AR-FL/AR-Vs co-regulators lead to prostate cancer as well as CRPC via the abnormal function of co-regulators on modulation of $A R$ transcriptional network [11-14]. AR-V7 is, to date, considered to be the putative AR variant. Importantly, $\mathrm{AR}-\mathrm{V} 7$ is rarely expressed in primary prostate cancer, but the expression of AR-V7 is increased in CRPC, serving as an independent predictive factor for CRPC development and cancer specific survival [15-17]. AR-V7 generation has generally been attributed to alternative splicing of $A R$ pre-mRNA and/or $A R$ genomic structural rearrangement $[3,4,18,19]$. Thus, to clarify the mechanisms involved AR-V7 generation as well as AR-FL/AR-V7 co-regulators would be crucial for understanding prostate cancer and the process of CRPC transformation.

In eukaryotic cells, mature messenger RNAs are formed by splicing from nascent precursor messenger RNAs, which is operated by spliceosomes [6]. Spliceosomal proteins can be aberrant expressed or somatic mutated in many cancers, and play vital roles in cancer development and progression [20-22]. Pre-mRNA processing factor 6 (PRPF6) is a component of spliceosome, and plays a role in the formation of spliceosome [23]. The study concerning the function of PRPF6 in tumor is rarely reported. It has been demonstrated that PRPF6 drives cancer proliferation by preferential splicing of genes associated with cell growth in colorectal carcinoma [24]. In addition to the function of PRPF6 on splicing, our study has previously demonstrated that PRPF6 interacts with the N-terminus of $A R$ and enhances AR-mediated transactivation [25]. However, little work has been done to understand the molecular mechanism underlying the modulation function of PRPF6 on AR action and the role of PRPF6 in prostate cancer.

Here, our results have demonstrated PRPF6 acts as a key regulator for action of both AR-FL and AR-V7, thereby participating in the enhancement of AR-FL and AR-V7-induced transactivation in prostate cancer. In addition, PRPF6 is recruited to cisregulatory elements in AR target genes and associates with JMJD1A to enhance AR-induced transactivation. PRPF6 also promotes expression of AR-FL and AR-V7. Moreover, PRPF6 depletion reduces tumor growth in prostate cancer-derived cell lines and results in significant suppression of xenograft tumors even under castration condition in mouse model. Furthermore, PRPF6 is obviously highly expressed in human prostate cancer samples. Taken together, our results demonstrate that PRPF6 is involved in enhancement of oncogenic AR signaling, which provides an insight to potential therapeutic strategies for prostate cancer, especially for CRPC.

\section{Materials and Methods}

\section{Plasmids}

Luciferase reporter plasmids and expression plasmids of Myc-tagged PRPF6, AR-FL and AR-V7 were described previously $[13,25]$. For FLAG-tagged full length and truncated mutants of PRPF6, the encoding cDNAs were cloned into plasmid vector pcDNA3-FLAG.

\section{Antibodies and reagents}

The antibodies used in this study were: antiPRPF6 (Bethyl, A302-773A; Santa Cruz, sc-166889; Proteintech, 23929-1-AP), anti-AR (Invitrogen, MA513426; Proteintech, 22089-1-AP), anti-MLL1 (Bethyl, A300-37A), anti-JMJD1A (Proteintech, 12835-1-AP), anti-PSA (Proteintech, 10679-1-AP), anti-FASN (Proteintech, 10624-2-AP), anti-UBE2C (Proteintech, 66087-1-lg), anti-H3K4me3 (Sigma-Aldrich, 05-745R), anti-H3K9me2 (Sigma-Aldrich, 05-1249), antiH3K36me3 (Millipore, ABE435), anti-FLAG (Shanghai Genomics, GNI4110-FG), anti-Myc (Shanghai Genomics, GNI4110-MC), anti- $\beta$-Actin (Proteintech 60008-1-Ig), anti-GAPDH (ABclonal, AC033). Other reagents used in this study were: normal mouse IgG (Santa Cruz, sc-2025), normal rabbit IgG (Santa Cruz, 2027). Transfections of plasmids and siRNAs were performed according to manufacturer's instructions of jetPRIME reagents (Polyplus-transfection). Charcoal/dextran-stripped serum (CSS) was prepared from fetal bovine serum (FBS, CLARK) according to the standard protocol.

\section{Cell culture}

Human PCa cell line LNCaP, CWR22Rv1 and VCaP were obtained from Kunming cell bank of Chinese Academy of Sciences, and were identified using PCR-STR analysis. LNCaP, CWR22Rv1 and VCaP cells were cultured in Roswell Park Memorial Institute medium 1640 (RPMI-1640, Gibco) with 10\% (v/v) FBS (CLARK). HEK293, cells were cultured in Dulbecco's modified Eagle medium (DMEM, Gibco), and supplemented with 10\%FBS. LNCaP-AI cells were cultured in media with $5 \%$ CSS. For the experiments with DHT stimulation, cells were cultured in medium with $5 \%$ CSS. Cells were incubated in a humidified incubator containing $5 \%$ $\mathrm{CO} 2$ at $37^{\circ} \mathrm{C}$.

\section{siRNA and lentiviral production}

For RNA interference (RNAi), chemically synthesized small interfering RNAs siRNA duplexes were purchased from Sigma (Sigma-Aldrich). The 
sequences of siRNAs targeting PRPF6 were: siPRPF6 1\# 5'-GAGAAGATTGGGCAGCTTA-3', siPRPF6 2\# 5'-GAUCUAAAUGACACCAAUU-3'. The sequence of negative control siRNA (siCtrl) was 5'- UUCUCCG AACGUGUCACGUTT -3'. For lentivirus-delivered RNAi, lentiviral productions targeting the same sequence as siPRPF6 1\# were purchased from Shanghai GeneChem Company and performed following manufacturer's instructions.

\section{Dual-luciferase reporter assays}

In dual-luciferase reporter assays, cells were co-transfected with the listed constructs. After $24 \mathrm{hrs,}$ cells harvested and lysed in passive lysis buffer. Luciferase activities were analyzed by dual-luciferase reporter assay system using GloMax Multi Jr detection system (Promega). Firefly luciferase activity was normalized to the activity of control renilla luciferase. Data represent the mean \pm SD from three experiments.

\section{Immunoprecipitation and western blot}

For immunoprecipitation, equal protein amounts of the extracted whole cell lysates were immunoprecipitated with specific antibody or control IgG. The immunoprecipitated complexes were analyzed by western blot.

\section{Immunofluorescence stain}

Cells were fixed by paraformaldehyde and blocked in donkey serum albumin. Then cells were incubated with primary antibody and subsequently FITC- or Cy3-conjugated secondary antibody (Jackson ImmunorRsearch Labs Inc). Nuclei were stained with DAPI (Roche).

\section{RNA isolation and quantitative real-time polymerase chain reaction (real-time $\mathbf{P C R}$ )}

Total RNA was extracted using TRIzol reagent (Thermo Fisher Scientific). cDNA synthesis was carried out with PrimeScript RT reagent kit with gDNA Eraser (TaKaRa), and then analyzed by SYBR Premix Ex Taq kit (TAKARA) according to the manufacturer's instructions in LightCycler96 system (Roche). Relative expression of genes was normalized by $18 \mathrm{~S}$ rRNA. Data represent the mean \pm SD of triplicate real-time PCR. Sequence of primers were list ed in Supplementary Table S1.

\section{RNA immunoprecipitation (RIP) and chromatin immunoprecipitation (ChIP)}

RIP experiments were performed using control IgG or antibodies using RIP assay kit (Millipore, Magna RIP kit) according to manufacturer's protocol. The immunoprecipitated RNA were converted to cDNA and quantified by real-time PCR. ChIP experiments were performed as previously described [13]. The purified immunoprecipitated DNA was quantified by real-time PCR. Results were shown as percentage of input. Data represent the mean \pm SD from three experiments. Sequence of primers were listed in Supplementary Table S2.

\section{Cell growth analysis and colony formation assays}

For growth curve assays, cells were seeded into 96-well plates at indicated densities. After indicated times, viability of cells were evaluated using the MTS assay (Promega) with the absorbance at $490 \mathrm{~nm}$. For colony formation assays, cells were maintained in medium for indicated times, then cells were fixed with paraformaldehyde and stained with coomassie blue dye.

\section{Xenograft tumor growth assays}

Animal works were approved and supervised by the Animal Ethics Committee of China Medical University. $5 \times 10^{5}$ cells were suspended in $100 \mathrm{ul}$ medium with half Metrigel (BD Biosciences) and injected subcutaneously into the 6-week-old male NOD/SCID mice with or without castration operation. Tumor size were measured by electronic caliper and the volume were calculated according to the formula: Volume $=(\Pi / 6) \times(\text { width } \times \text { length })^{3 / 2} .21$ or 27 days after inoculation, mice were killed in keeping with the policy of the humane treatment of tumor bearing animals.

\section{Immunohistochemical (IHC) analysis and clinical prostate cancer samples}

Formalin-fixed paraffin-embedded sections of PCa tissues and adjacent non-cancerous tissues were obtained from the first hospital of China Medical University. Ethical approval for sample collection and tissue use was approved by the Human Research Ethics Committee of China Medical University. A total of 162 tissues, including 20 PCa tissues without matched adjacent non-cancerous tissues, and 71 pairs of PCa and matched adjacent non-cancerous tissues, were collected to perform IHC staining. IHC experiments were performed as previously described [12]. The immunohistochemical stain of PRPF6 expression was evaluated by the Hscore method, as previously described [26]. Briefly, the intensity was scored as follows: 0 , negative; 1 , weak; 2 , moderate and 3 , strong. The final score, which ranged from 0 to 3 , was determined by summing the intensity score multiplied by the corresponding proportion.

\section{Statistical analysis}

To determinate the significant difference in results of real-time PCR, dual-luciferase reporter 
assays and xenograft tumor growth assays, Student's $t$-test was used with Prism Graphpad program. SPSS software was used to analyze results of IHC. Student's $t$-test was used to determinate the significant difference between immunohistochemical expression of PRPF6.

\section{Results}

\section{PRPF6 promotes cell growth in prostate cancer-derived cell lines}

To investigate the physiological role of PRFP6 in prostate cancer, we examined the endogenous protein expressions of PRPF6 in four cultured prostate cancer-derived cell lines as indicated (Figure 1A). These cells generally expressed PRPF6 protein. In three AR-positive prostate cancer cell lines, PRPF6 was lowly expressed in LNCaP cells that expressed AR-FL but not AR-Vs. In CWR22Rv1 cells and VCaP cells, which constitutively carry AR-FL and AR-Vs, PRPF6 were significantly highly expressed. As we previously reported that PRPF6 enhances transactivation function of AR [25], we then analyzed the impact of PRPF6 on the growth and motility characteristics of AR-positive prostate cancer cells by lentivirus-mediated knockdown of PRPF6 (Supplementary Figure S1). Colony formation experiments were performed in the absence and presence of androgen (DHT), and results showed PRFP6 knockdown impaired cell ability to form colonies in CWR22Rv1 cells (Figure 1B). The same trend was showed in LNCaP cells with lower ability of colonies formation (Supplementary Figure S2). Consistently, cell growth with PRFP6 knockdown was slower than control cells, in both CWR22Rv1 (Figure 1C) and LNCaP cells (Figure 1D). Further, exogenous expression of AR rescued the growth inhibition by PRPF6 knockdown in CWR22Rv1 cells, indicating that growth promotion by PRPF6 is associated with AR (Figure 1E).

In addition, we performed in vivo tumor growth analysis in a mouse xenograft model with CWR22Rv1 cells. Results showed that PRPF6 knockdown reduced tumor burden, with smaller volumes and slower growth rate of the xenograft tumors (Figure $1 \mathrm{~F}$ and $1 \mathrm{G})$. In line with the tumor growth curve, the tumor weights of cells with PRPF6 knockdown were significantly lower (Figure 1H). Consistent with the AR-related growth promoting function of PRPF6, xenograft tumors derived by PRPF6 knockdown cells exhibited a significant reduction in prostate-specific antigen (PSA) and ubiquitin-conjugating enzyme E2 C (UBE2C) levels, which are target genes of AR (Figure 1I). Taken together, the above results indicated that PRPF6 promotes cell proliferation of prostate cancer cells in vitro and in vivo.

\section{PRPF6 associates with AR in prostate cancer cells}

We then wonder to test the association between PRPF6 and AR in prostate cancer cells. Co-IP results showed that exogenous expressed AR-FL was precipitated with Myc-tagged PRPF6 in HEK293 cells, both in the absence and presence of DHT (Figure 2A). Further, in CWR22Rv1 cells, the interaction between endogenous PRPF6 and AR-FL could be observed (Figure 2B and 2C). In addition to AR-FL, PRPF6 also interacted with endogenous $\mathrm{AR}-\mathrm{Vs}$ in CWR22Rv1 cells (Figure 2C). Moreover, results of immunofluorescence experiments showed that endogenous PRPF6 located in the nucleus in prostate cancer cells including LNCaP, CWR22Rv1, and DU145 (Supplementary Figure S3A-S3C). In LNCaP cells, PRPF6 was compartmentalized in the nucleus with AR under DHT treatment (Supplementary Figure S3A). In CWR22Rv1 cells, PRPF6 was distributed together with AR in the nucleus, both in the absence and presence of DHT (Supplementary Figure S3B). These data indicated that PRPF6 physically associates with AR in prostate cancer cells.

PRPF6 protein contains an N-terminal domain followed by multiple tetratricopeptide repeat (TPR) motifs. In addition, we generated expression plasmids encoding PRPF6 truncated mutants (Figure 2D and Supplementary Figure S4). Our results of immunofluorescence experiments showed that PRPF6 C (309-941 aa) localized in both nucleus and cytoplasm (Figure 2E). Meanwhile, PRPF6 N (1-308 aa), PRPF6 full length (FL), PRPF6 5TPR (1-482 aa), PRPF6 10TPR (1-640 aa) and PRPF6 15TPR (1-809 aa) showed nuclear localization (Figure 2E). And the localizations of PRPF6 5TPR (1-482 aa) and PRPF6 10TPR (1-640 aa) were almost identical to PRPF6 FL, whereas PRPF6 15TPR (1-809 aa) showed obvious speckle localization (Figure 2E).

\section{PRPF6 coactivates AR-FL or AR-V7-mediated transactivation in prostate cancer cells}

We next performed a series of luciferase assays to investigate the function of PRPF6 on AR-mediated transcription. By employing three luciferase reporter systems, including ARE-luc, MMTV-tk-luc and PSA-tk-luc reporters, we demonstrated that PRPF6 significantly enhanced AR-FL-mediated transactivation in the presence of DHT (Figure 3A-3C). Further, PRPF6 significantly up-regulated AR-V7mediated transcriptional activity in the absence of DHT (Figure 3A). Moreover, luciferase assays were performed with these truncated mutants. PRPF6 5TPR and PRPF6 10TPR showed similar ability to enhance 
AR-FL-mediated transactivation as PRPF6 FL, while nor PRPF6 N or PRPF6 $\mathrm{C}$ has obvious effect (Figure 3D). Intriguingly, PRPF6 15TPR showed significant enhancement of AR-FL-mediated transactivation (Figure 3D). In CWR22Rv1 cells, PRPF6 significantly enhanced the transcriptional activity of ARE-luc reporter gene with or without DHT treatment, which represents the endogenous AR-FL and AR-Vsmediated transactivation (Figure $3 \mathrm{E}$ ). Based on the above results, we conclude that PRPF6 functions as a coactivator of AR-FL, and enhances AR-V7 action in the absence of DHT.

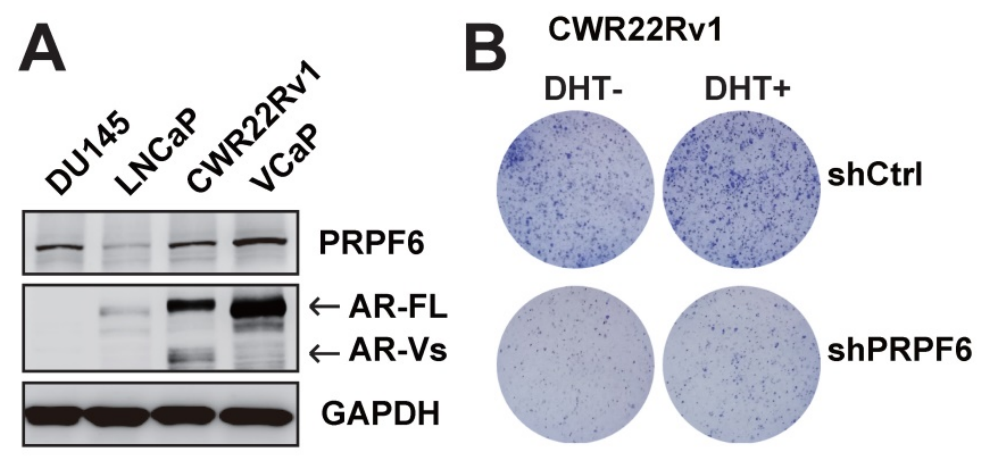

\section{LNCaP}

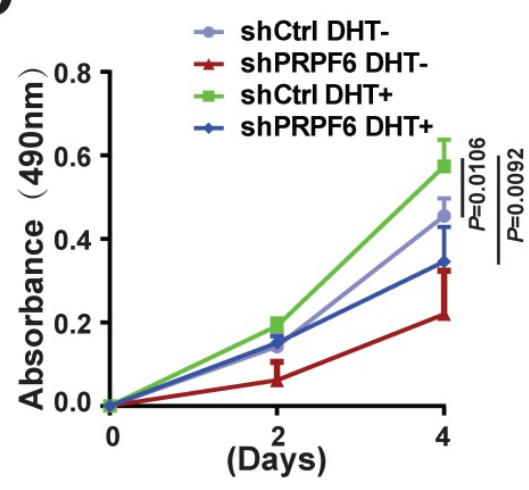

$\mathbf{F}$
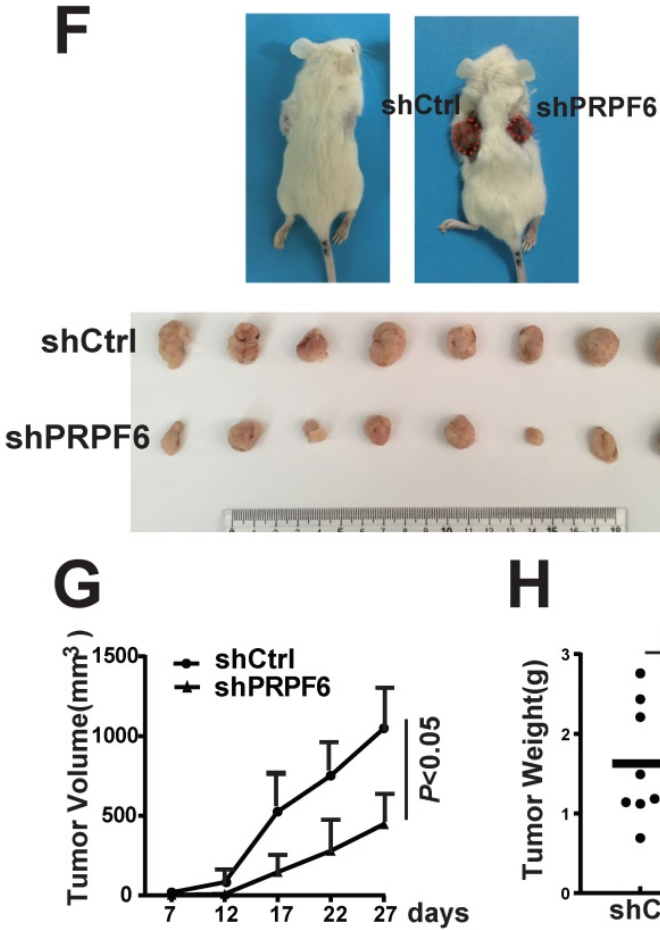

H

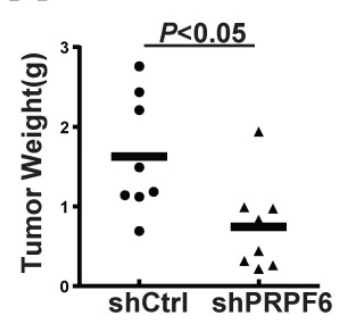

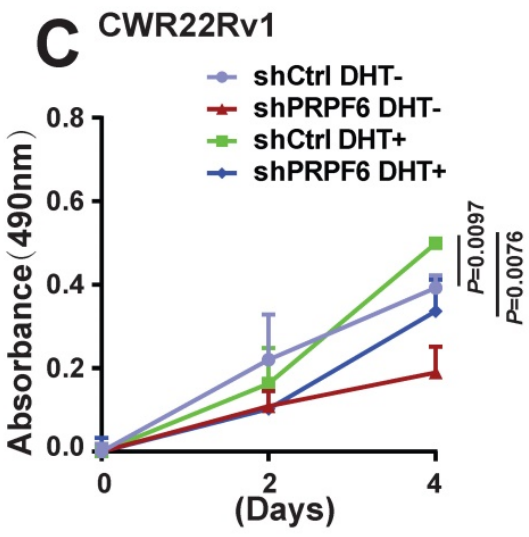

E CWR22Rv1
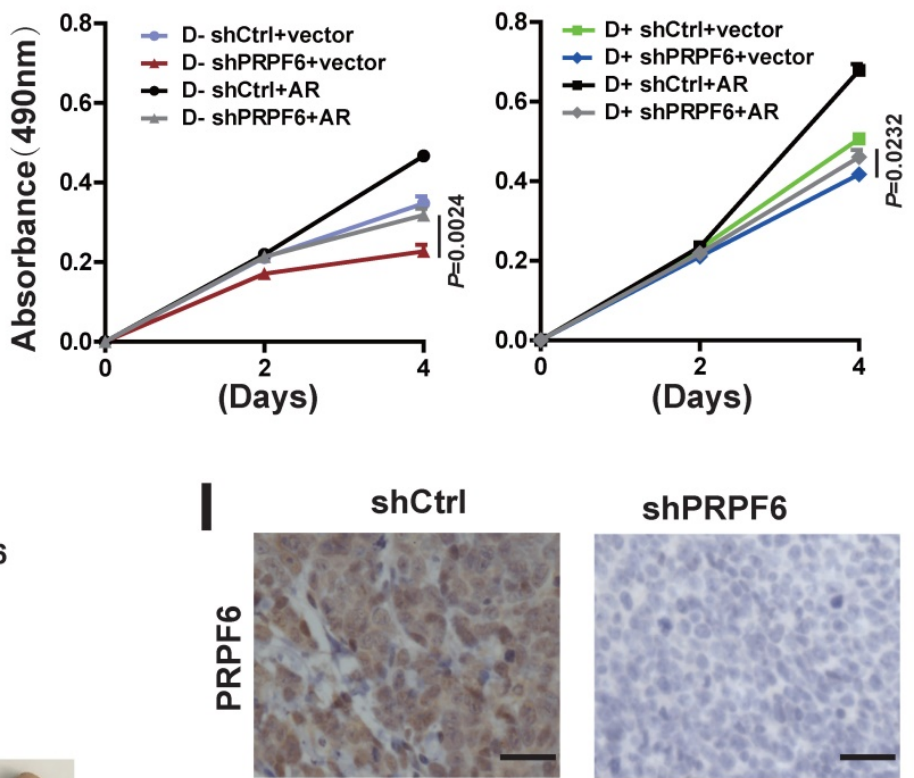

shPRPF6
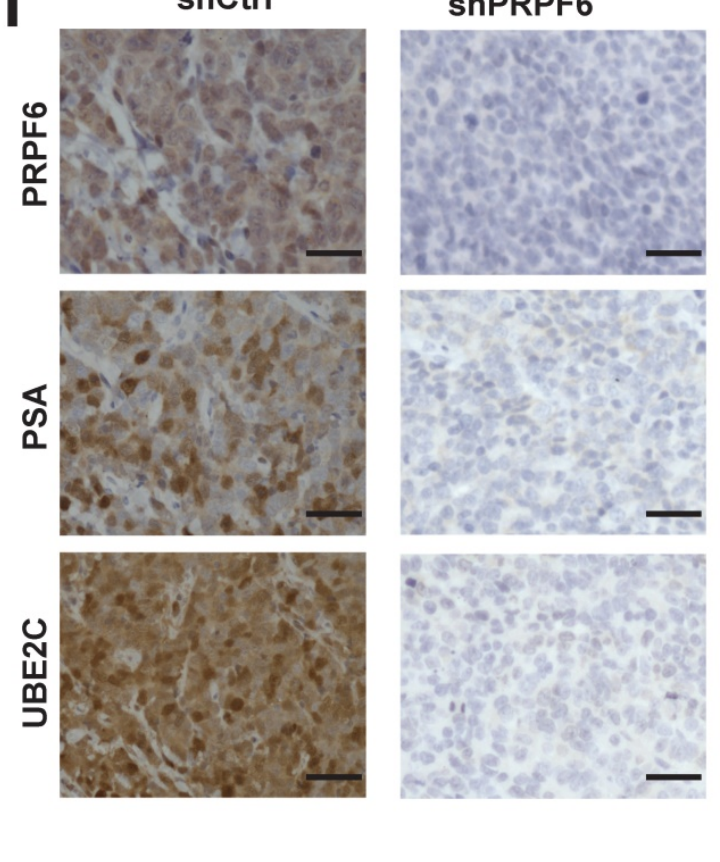

Figure 1. PRPF6 promotes growth of prostate cancer cells. A. PRPF6 and AR expressions in prostate cancer-derived cell lines were analyzed by western blot. Protein extractions of different cell lines were subjected to western blot analysis using antibodies against PRPF6 (Proteintech) and AR (Proteintech). GAPDH was used as internal control. 
B. Effects of PRPF6 knockdown on the colony formation of CWR22Rv1 cells were shown. CWR22Rv1 cells (1000 per well) were infected with lentivirus shRNA targeting PRPF6 (shPRPF6) or control lentivirus (shCtrl), and then were treated with $10^{-8} 8 \mathrm{M} \mathrm{DHT}$ or ethanol for 14 days. C and D. PRPF6 knockdown repressed cell proliferation. CWR22Rv1 cells (C) and LNCaP (D) (2500 per well) with shPRPF6 or shCtrl were treated with 10-8 M DHT or ethanol. After indicated time, MTS reagent was added, then absorbance at $490 \mathrm{~nm}$ was measured and plotted. Data were means \pm SD of three independent experiments. Student's $t$-test was performed. E. Growth inhibition of prostate cancer cells by PRPF6 was associated with AR. CWR22Rv1 cells ( 2500 per well) with shPRPF6 or shCtrl were transfected with AR expression lentivirus or control lentivirus, and then were treated with $10^{-} 8 \mathrm{MDHT}$ (right) or ethanol (left). After indicated time, MTS reagent was added, then absorbance at $490 \mathrm{~nm}$ was measured and plotted. Data were means \pm SD of three independent experiments. Student's $t$-test was performed. F. Representative photograph of mice with tumor xenografts (upper panel). Photograph of all xenograft tumors derived from CWR22Rv1 cells with shPRPF6 or shCtrl (lower panel). G. Tumor volumes of xenografts after inoculation were shown. The volume of xenograft tumors derived from CWR22Rv1 cells with shPRPF6 or shCtrl were measured every 5 days. Data represent means \pm SD. Student's $t$-test was performed. H. Final tumor weights of xenograft tumors derived from CWR22Rvl cells with shPRPF6 or shCtrl were measured and shown. Horizontal lines represent the mean values. Student's $t$-test was performed. I. Expressions of indicated proteins in xenograft tumors were detected by IHC assays. Scale bars, $100 \mu \mathrm{m}$.

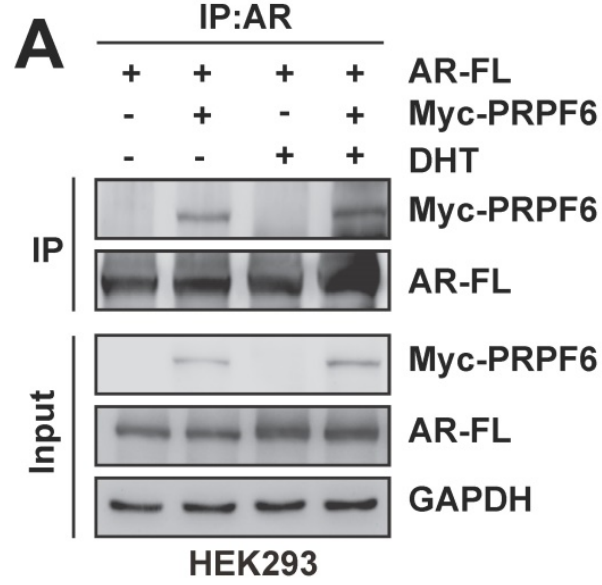

B
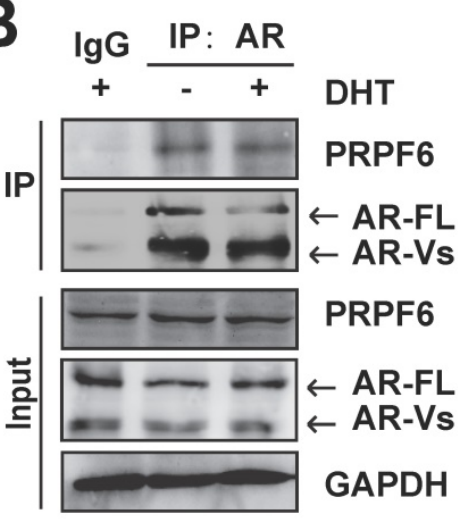

PRPF6

CWR22Rv1

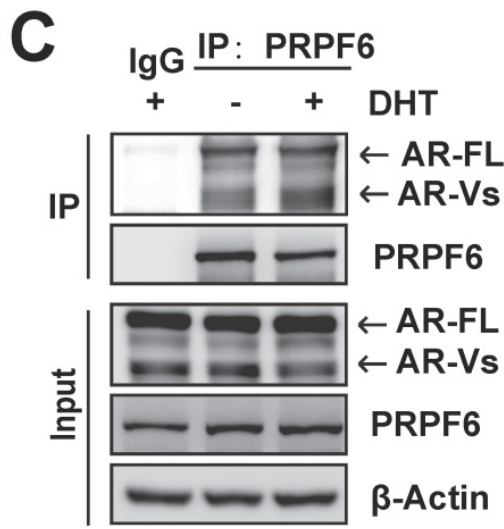

CWR22Rv1

GAPDH
D

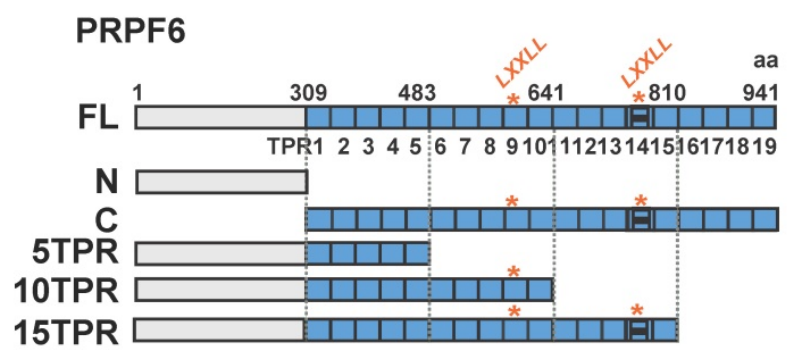

E

FLAGAR PRPF6
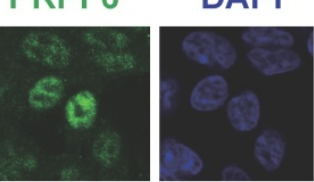

MERGE DHT

FL
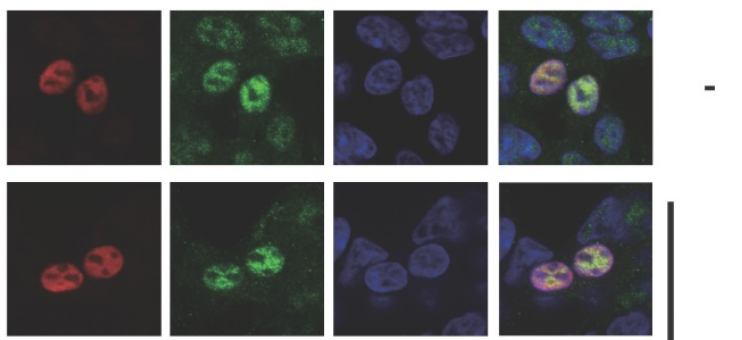

$\mathbf{N}$
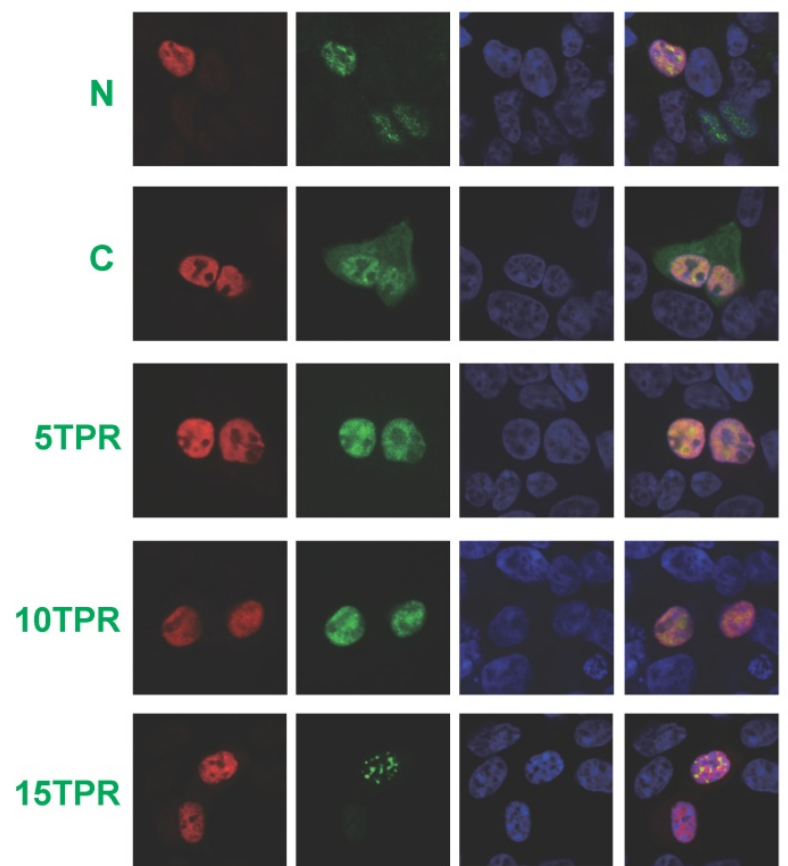

HEK293

Figure 2. PRPF6 interacts with AR in prostate cancer cells. A. Exogenous PRPF6 interacted with AR-FL in HEK293 cells. HEK293 cells were co-transfected with plasmids expressing AR-FL and PRPF6 or vector as indicated. After 4 hrs, cells were treated by DHT (10-8 M) or ethanol vehicle for another 24 hrs. Then cells were harvested and equal amounts of cell lysates were subjected to immunoprecipitation with anti-AR antibody (Invitrogen). The immunoprecipitated proteins were subjected to western blot analysis using anti-AR (Proteintech) and the indicated antibodies. GAPDH was used as internal control. B and C. Endogenous PRPF6 and AR interacted with each other in 
CWR22Rv1 cells. Upon treatment of $10^{-8} \mathrm{M}$ DHT or ethanol vehicle for 24 hrs, CWR22Rv1 cells were harvested and equal amounts of cell lysates were subjected to immunoprecipitation with normal IgG, anti-AR antibody (Invitrogen) or anti-PRPF6 antibody (Santa Cruz). The immunoprecipitated proteins were subjected to western blot analysis using antibodies against PRPF6 (Proteintech) and AR (Proteintech). GAPDH or $\beta$-Actin was used as internal control. D. Diagram of plasmids encoding FLAG-tagged PRPF6-FL and truncated mutants. E. Subcellular localizations of the AR and PRPF6-FL or truncated mutants. HEK293 cells were co-transfected with plasmids expressing AR-FL and PRPF6-FL or truncated mutants. After 10-8 M DHT stimulation or ethanol vehicle for $4 \mathrm{hrs}$, cells were fixed and stained with antibody against FLAG and AR (Proteintech). DAPI was used to visualize the nucleus (blue). Merged images were shown as indicated.
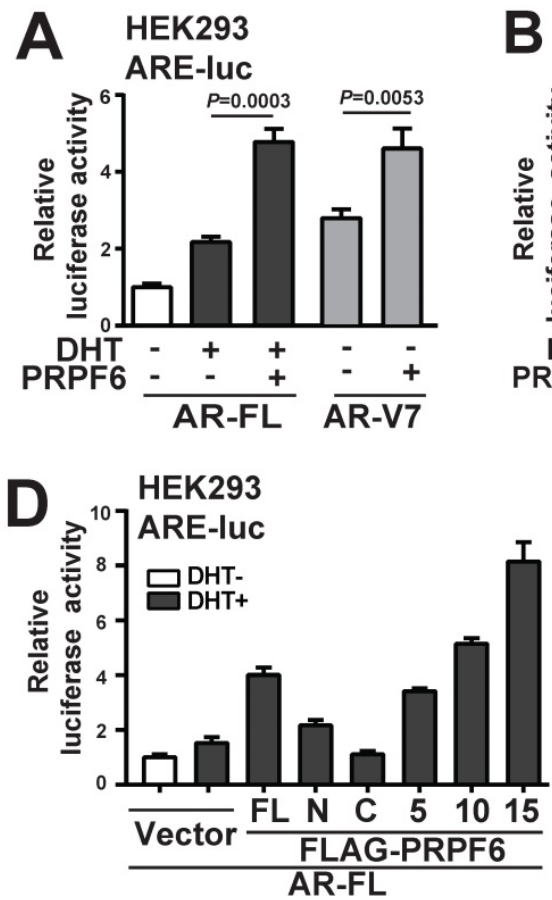
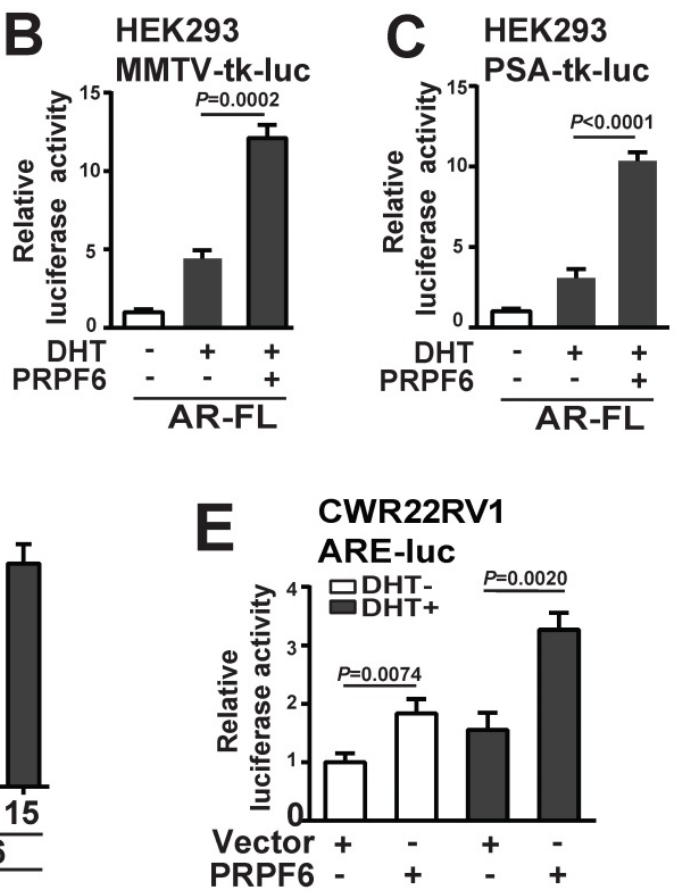

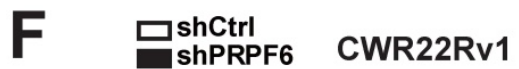

PRPF6

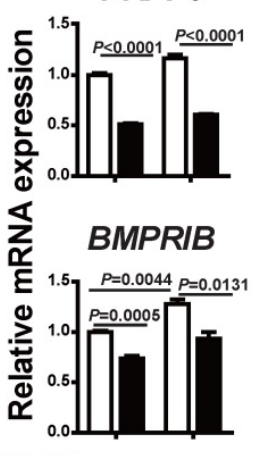

DHT
PSA

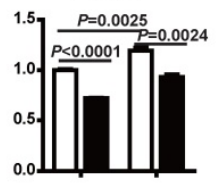

ELOVL5

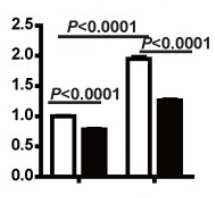

KLK2

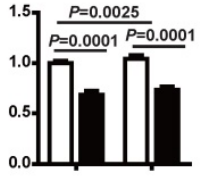

HPGD

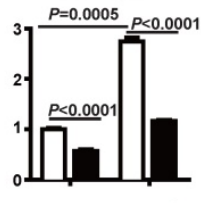

FASN

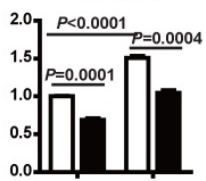

SLC45A3

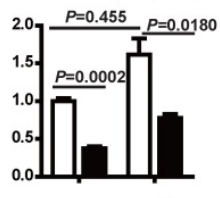

TMPRSS2

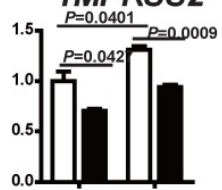

ACPP

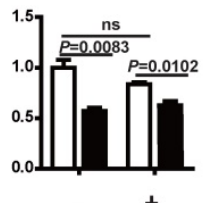

UBE2C

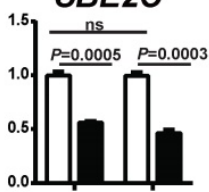

AR-FL

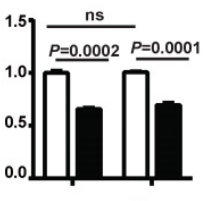

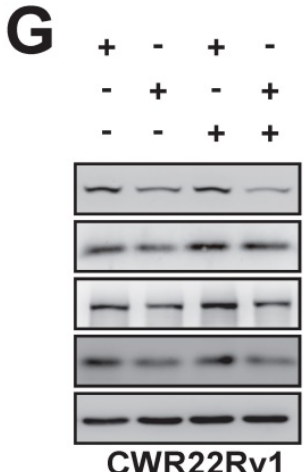

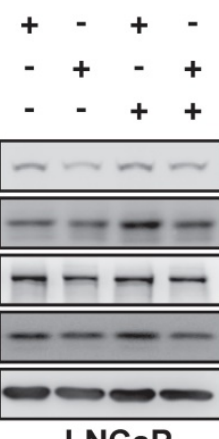

shCtrl

shPRPF6

DHT

PRPF6

PSA

FASN

UBE2C

GAPDH

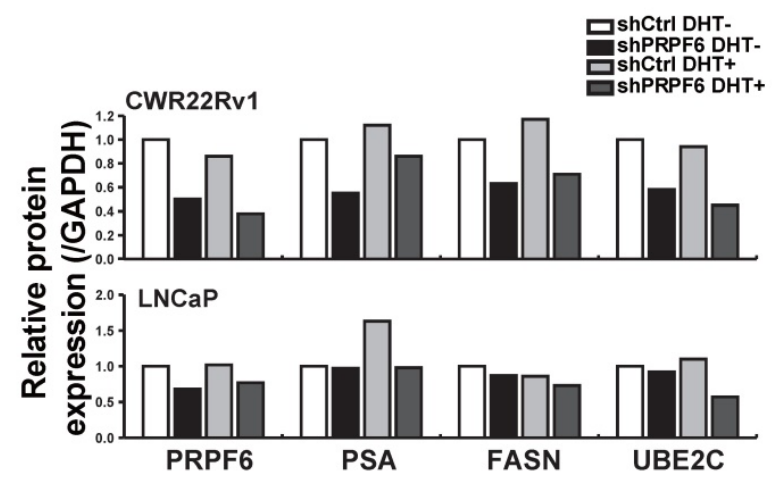

Figure 3. PRPF6 promotes AR-FL and AR-V7-mediated transactivation. A, B and C. PRPF6 enhanced AR-FL and AR-V7-mediated transactivation. HEK293 cells were co-transfected with AR-FL (A, B and C) or AR-V7 (A) and PRL-TK, together with the indicated reporter luciferase plasmids. After 4 hrs, cells were treated by DHT (10-8 M) or ethanol vehicle for another 24 hrs. Then, cells were lysed and assayed using the dual-luciferase reporter assay system. Student's $t$-test was performed. D. Effects of 
PRPF6-FL or truncated mutants on AR-FL-mediated transactivation. HEK293 cells were co-transfected with and ARE-luc, PRL-TK, together with AR-FL and indicated plasmids. After 10-8 M DHT stimulation or ethanol vehicle for $24 \mathrm{hrs}$, cells were lysed and assayed using the dual-luciferase reporter assay system. E. PRPF6 enhanced endogenous AR-mediated transactivation in CWR22Rv1 cells. CWR22Rv1 cells were co-transfected with and ARE-luc, pRL-TK, together with PRPF6 or vector plasmids as indicated. After $4 \mathrm{hrs}$, cells were treated by DHT (10-8 M) or ethanol vehicle for another $24 \mathrm{hrs}$. Then, cells were lysed and assayed using the dual-luciferase reporter assay system. Student's $t$-test was performed. F. Effect of PRPF6 knockdown on the mRNA expression of AR target genes and AR-FL in CWR22Rv1 cells. Cells were infected with shPRPF6 or shCtrl. After treatment of 10-8 M DHT or ethanol vehicle for $24 \mathrm{hrs}$, cells were collected for RNA extraction and quantitative real-time PCR were performed. Student's $t$-test was performed. ns, not significant. G. Effect of PRPF6 knockdown on the protein expression of AR target genes in CWR22Rv1 and LNCaP cells. CWR22Rv1 (left) or LNCaP (right) cells with shPRPF6 or shCtrl were treated with 10-8 M DHT or ethanol vehicle for 24 hrs, then cells were harvested for protein extraction. Western blot was performed to detect the expression of proteins using anti-PRPF6 (Santa Cruz) and the indicated antibodies. GAPDH was used as internal control. The protein expressions were quantified by densitometry and shown in the right panel, with GAPDH as the reference.

Having demonstrated that PRPF6 functions as a cofactor of AR-FL and AR-V7, we next examined whether PRPF6 knockdown led to altered expression of endogenous AR target genes. To this end, we first turn to LNCaP cells, which is androgen-sensitive and without AR-V7 expression. We evaluated the mRNA expression of a panel of well-characterized AR targets, that include PSA, kallikrein related peptidase 2 (KLK2), fatty acid synthase (FASN), transmembrane protease serine 2 (TMPRSS2), UBE2C, bone morphogenetic protein type $I B$ receptor (BMPRIB), ELOVL fatty acid elongase 5 (ELOVL5), 15-hydroxyprostaglandin dehydrogenase (HPGD), solute carrier family 45 a3 member (SLC45A3), and acid phosphatase 3 (ACPP). Rea-time PCR results showed that lentivirus-mediated PRPF6 knockdown resulted in a significant decrease in the expression of 9 AR target genes, including PSA, KLK2, FASN, TMPRSS2, UBE2C, BMPRIB, HPGD, SLC45A3 and $A C P P$ with DHT treatment in LNCaP cells (Supplementary Figure S5). Meanwhile, we analyzed the impact of PRPF6 knockdown in CWR22Rv1 cells, and found expressions of the 10 genes were all repressed both with and without DHT treatment (Figure 3F). Interestingly, PRPF6 knockdown suppressed expression of AR-FL (Supplementary Figure S5 and Figure 3F). Moreover, western blot analysis of the endogenous protein expression in LNCaP cells confirmed that knockdown of PRPF6 led to obvious reductions of PSA protein levels under DHT treatment, while repressed FASN and UBE2C protein expressions both with and without DHT treatment (Figure 3G). In CWR22Rv1 cells, PSA, FASN and UBE2C protein expressions were downregulated by PRPF6 knockdown in the absence or presence of DHT, respectively (Figure 3G). Taken together, these data suggested that PRPF6, as a cofactor, is required for endogenous AR-regulated gene activation.

\section{PRPF6 associates with JMJDIA to enhance AR-induced transactivation}

We next investigated molecular mechanisms underlying the modulation of PRPF6 on AR-mediated transcription. AR binds to cognate AR-responsive elements (AREs) on its target genes to induce target gene transcription upon the treatment of androgen. We thus turn to examine the recruitment of PRPF6 or
AR on the cis-regulatory elements of target genes with ChIP assay. The results demonstrated that AR or PRPF6 was recruited to AREI/II region in the promoter of PSA gene (Figure 4A). A growing body of evidence support that coordination of pre-mRNA splicing with chromatin remodeling events are involved in transcriptional regulation [27-29], we then ask whether PRPF6 would influence some epigenetic events, including histone modification. Histone H3K4me and H3K36me are hallmarks of actively transcribed euchromatin, whereas $\mathrm{H} 3 \mathrm{~K} 9 \mathrm{me}$ is associated with repressive heterochromatin state. We then tested the alteration of histone H3K9me2, H3K4me3, or H3K36me3 at chromatin encompassing the cis-regulatory elements in the cells with knockdown of PRPF6. Interestingly, our results showed that PRPF6 depletion increased histone H3K9me2 level and decreased H3K4me3 level, while alternation of histone H3K36me3 was not obvious (Figure 4A). Similar results were observed at ARE III region in PSA enhancer and ARE region in KLK2 promoter (Figure 4B and 4C). In addition, PRPF6 knockdown also decreased the recruitment of $\mathrm{AR}$ at the examined sites (Figure 4A-4C).

We then turn to ask whether PRPF6 associates with histone methyltransferase or demethylase to coactivate AR-mediated transactivation. It has been reported that JMJD1A, which is a histone H3K9 demethylase, acts as a coactivator for $\mathrm{AR}$ by epigenetic regulation of histone $\mathrm{H} 3 \mathrm{~K} 9$ me marks [30, 31]. The mixed-lineage leukemia 1 (MLL1), which is a histone methyltransferase of H3K4, could be recruited at ARE of AR target genes, subsequently increasing histone H3K4 trimethylation [13]. In order to determine whether PRPF6 might interact with JMJD1A or MLL1 to regulate AR-mediated transcription, Co-IP experiments were performed to examine the association of PRPF6 with JMJD1A or MLL1. The results demonstrated an interaction between PRPF6 and JMJD1A, but not MLL1 (Figure 4D). Moreover, AR interacts with PRPF6 together with JMJD1A, and knockdown of PRPF6 weakened the interaction between JMJD1A and AR (Figure 4E), which is in line with the alternation of histone H3K9me2 at ARE regions by PRPF6 knockdown. Since PRPF6 knockdown can reduce the expression of endogenous $A R$, we further detected the interaction 
between JMJD1A and AR under AR overexpressing condition. Similar to the result showed in Figure 4E, Co-IP experiment showed decreased interaction between JMJD1A and AR by PRPF6 knockdown (Figure 4F). Taken together, we speculate that PRPF6
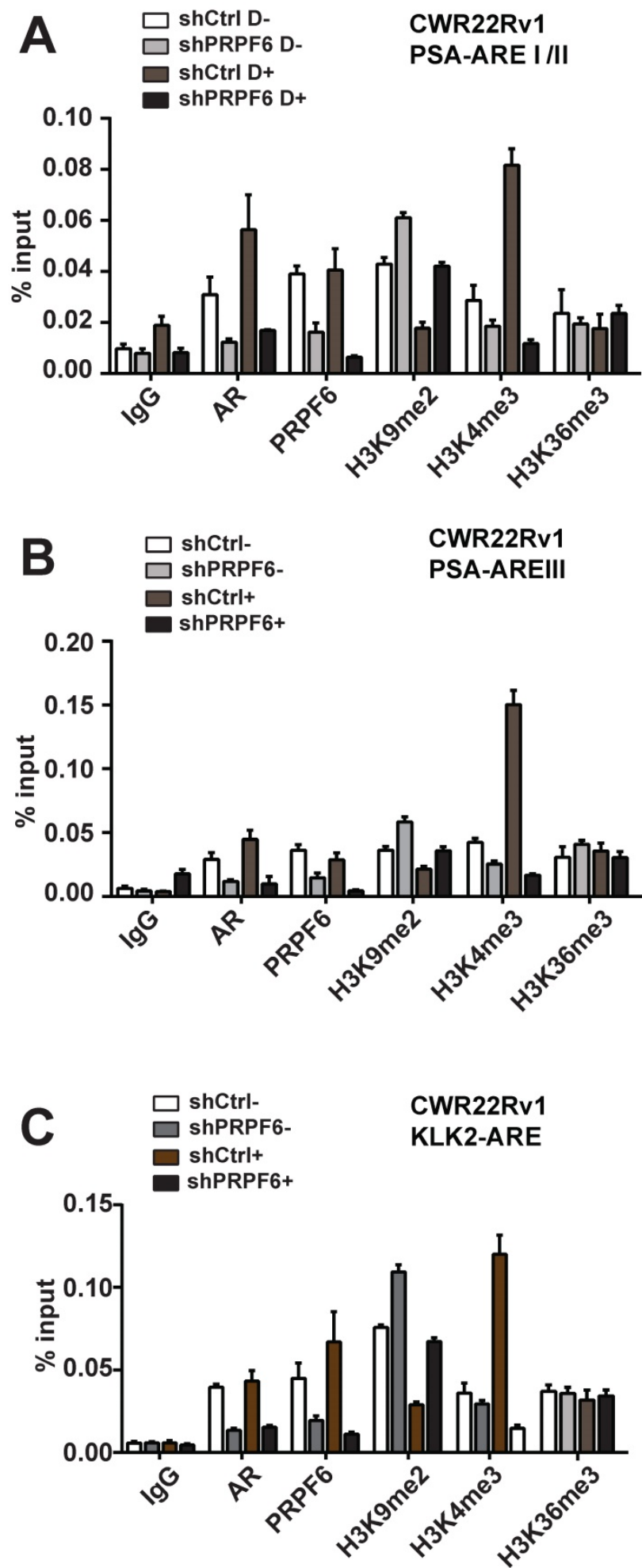

associates with JMJD1A, thereby altering the histone H3K9me2 level at the ARE regions of AR target genes to maintain the active chromatin state, subsequently promoting AR-mediated transcription.

D

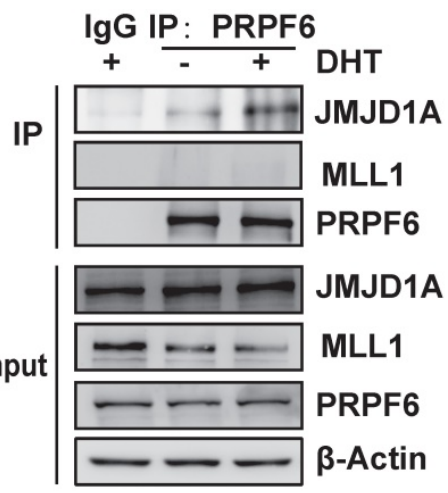

E

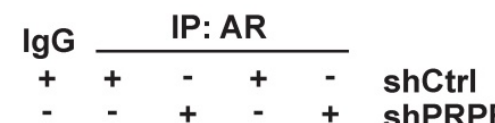

$-\quad+\quad+\quad+$ shPRPF6

$+\quad-\quad+\quad+$ DHT
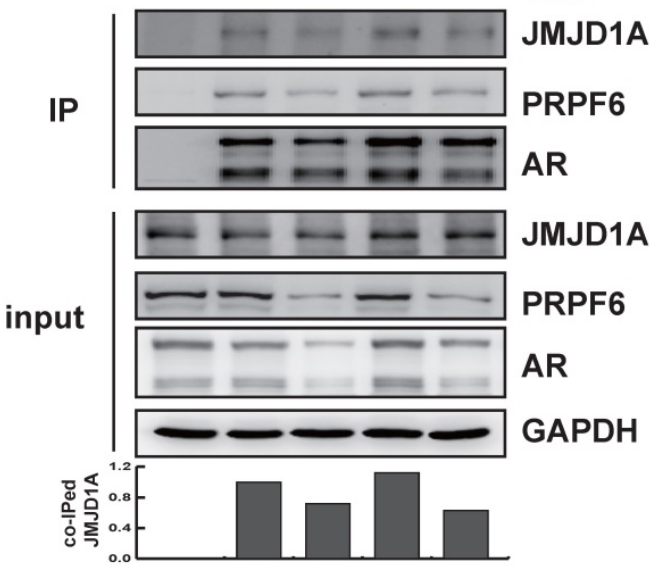

$\mathbf{F}$
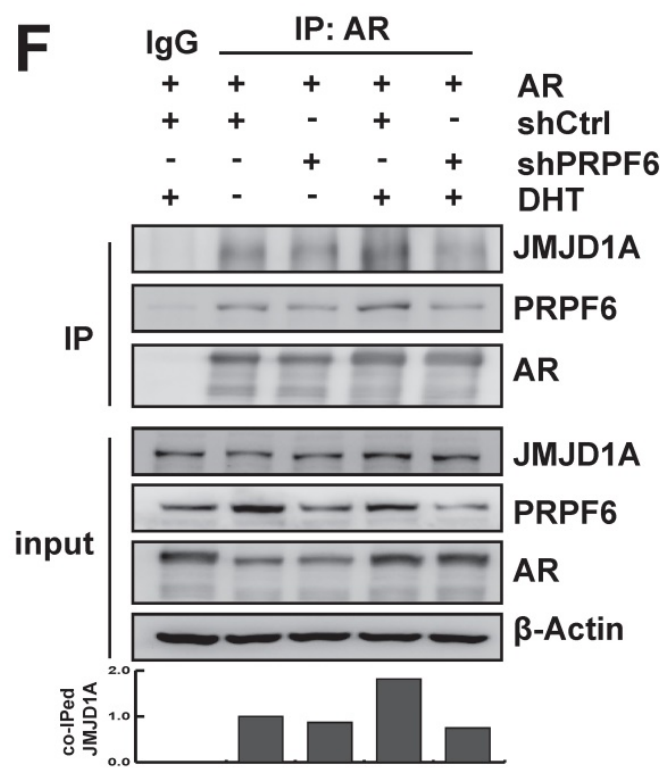

Figure 4. Mechanisms involved in promotion of AR-mediated transactivation by PRPF6. A, B and C. PRPF6 knockdown impacted on the recruitments of PRPF6 and $A R$, as well as the epigenetic modification on AREI/II (A), AREIII (B) in PSA and ARE (C) in KLK2. CWR22Rvl cells infected with shPRPF6 or shCtrl were treated with 10-8 M 
DHT or ethanol for 4 hrs, then cells were subjected to ChIP assays with using normal lgG, anti-AR (Invitrogen), anti-PRPF6 (Bethyl) or the indicated antibodies. D. PRPF6 interacts with JMJD1A in CWR22Rv1 cells. Upon treatment of $10^{-} 8 \mathrm{M} \mathrm{DHT}$ or ethanol vehicle for $24 \mathrm{hrs,} \mathrm{CWR22Rv1} \mathrm{cells} \mathrm{were} \mathrm{harvested} \mathrm{and} \mathrm{equal} \mathrm{amounts} \mathrm{of} \mathrm{cell} \mathrm{lysates} \mathrm{were}$ subjected to immunoprecipitation with normal IgG, anti-PRPF6 antibody (Proteintech). The immunoprecipitated proteins were subjected to western blot analysis using antibodies against PRPF6 (Proteintech), MLL1 (Bethyl) and JMJDIA (Proteintech). $\beta$-Actin was used as internal control. E and F. PRPF6 knockdown reduced the interaction between JMJD1A and AR. CWR22Rv1 cells infected with shPRPF6 or shCtrl were transfected with AR expression plasmid (in F) or not (in E). Then, upon treatment of 10-8 M DHT or ethanol vehicle for $24 \mathrm{hrs}$, cells were harvested and equal amounts of cell lysates were subjected to immunoprecipitation with normal lgG, anti-AR antibody (Invitrogen). The immunoprecipitated proteins were subjected to western blot analysis using antibodies against PRPF6 (Proteintech), JMJDIA (Proteintech) and AR (Proteintech). GAPDH was used as internal control. Co-immunoprecipitated (co-IPed) JMJDIA was quantified by densitometry and shown in the lower panel.

\section{PRPF6 knockdown attenuates AR and AR-V7 transcription}

It is noticeable that PRPF6 knockdown reduced the mRNA (Figure 3F and Supplementary Figure S5) and protein expression of AR (Figure 4E), indicating that PRPF6 might be involved in $A R$ itself transcription. In order to assess the effect of PRPF6 on endogenous expression of AR, we knocked down PRPF6 expression using two specific siRNAs targeting different sequences of PRPF6. In LNCaP cells, mRNA level of $A R-F L$ was reduced after PRPF6 knockdown (Figure 5A). In CWR22Rv1 cells, PRPF6 knockdown resulted in decreased mRNA levels of both $A R-F L$ and $A R-V 7$ (Figure 5B), which is the major form of AR-Vs in this cell line [32]. Similarly, knockdown of PRPF6 decreased the protein levels of both AR-FL and AR-Vs (Figure 5C), and ectopic expression of PRPF6 increased the protein expressions of both AR-FL and AR-Vs (Figure 5D) in CWR22Rv1 cells. Consistent with these results, both mRNA and protein levels of AR-FL as well as AR-V7 were significantly reduced upon lentivirus-mediated PRPF6 knockdown (Figure 5E-5H). We next assessed whether PRPF6 participates in alternative splicing of $A R-V 7$ in CWR22Rv1 cells. As shown in Supplementary Figure S6A, constitutively expressed $A R-F L$ mRNA consists of eight exons, whereas $A R-V 7$ mRNA only includes exons 1-3 and a cryptic exon $3 b$. We performed RIP experiment to identify potential PRPF6-binding regions using primers targeting the $\mathrm{P} 3 \mathrm{~b}$ region [32], and the results showed strong enrichment of PRPF6 on P3b region (Supplementary Figure S6B), indicating that PRPF6 may participates in AR-V7 alternative splicing.

It is reported that $A R$ could sustain its own expression by binding enhancers located upstream of the transcription start site in the absence of ligand [33]. We therefore evaluated PRPF6 recruitment on the reported AREM1 and AREM2 sites (Figure 5I) by ChIP assay using site-specific primers. Our data showed that PRPF6 was recruited at AREM1 site (Figure 5J), while AR was recruited at both AREM1 and AREM2 sites as reported, suggesting that PRPF6 may be involved in modulation of AR gene transcription. Taken together, these data indicated that PRPF6 promotes AR-FL and AR-V7 transcription and may play a role in transactivation of $A R$ gene.

\section{PRPF6 is required for growth of prostate cancer cells under androgen-depleted condition}

Both AR-FL and AR-V7 are necessary to support proliferation of CRPC cells under castration conditions [34]. We then set up to test whether PRPF6 is necessary for prostate cancer cell growth under androgen-depleted condition. We first investigated the PRPF6 function in long-term androgen deprivation cells. We generated an androgenindependent $\mathrm{LNCaP}$ subline (named $\mathrm{LNCaP}-\mathrm{AI}$ ) by culturing LNCaP cells in media containing androgendeprived charcoal/dextran-stripped serum (CSS) (5\%), which mimics androgen deprivation conditions, for about 6 months. The LNCaP-AI cells were able to proliferate under CSS (Supplementary Figure S7A) and showed a higher ability for colony formation (Supplementary Figure S7B). Similar to previous description [35], LNCaP-AI cells exhibited significant increased expression of AR-FL and AR-V7 (Supplementary Figure S7C and S7D). Consistent with the results in CWR22Rv1 cells, decreased expressions of AR-FL and AR-Vs after PRPF6 knockdown were observed in the LNCaP-AI cells (Figure 6A). In addition, knockdown of PFPF6 reduced colony formation and growth of LNCaP-AI cells under CSS culture conditions (Figure 6B and 6C).

To further validate the role of PRPF6 in tumor growth under castration conditions, we next performed in vivo xenograft assays in castrated male mice. As shown in Figure 6D, tumor formation from castration-resistant CWR22Rv1 cells was observed in castrated male mice, and knockdown of PRPF6 significantly suppressed xenograft tumor growth derived from CWR22Rv1 cells. Compared with control cells, PRPF6-knockdown cells showed slower growth rate, smaller volume and decreased tumor weight (Figure 6E and 6F). We also examined the staining of PSA and UBE2C in xenograft tumors, and the result showed that PSA and UBE2C levels were decreased in PRPF6 knockdown cell-derived xenograft tumors (Figure 6G). Taken together, these data suggested that PRPF6 promotes prostate cancer cell growth under androgen-depleted condition in vitro and in vivo. 
A LnCaP PRPF6

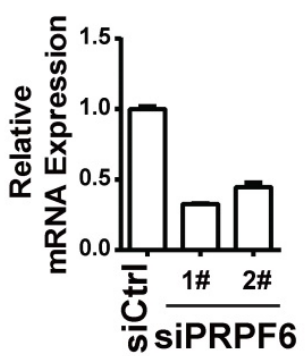

AR-FL

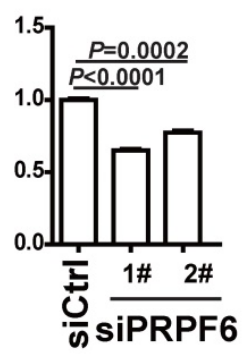

B CWR22Rv1

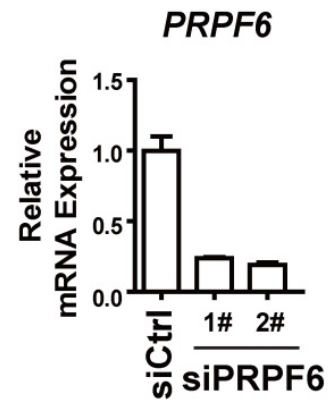

AR-FL

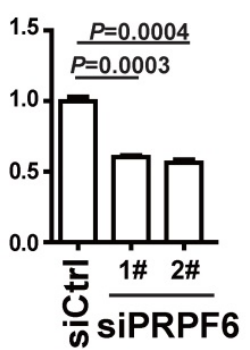

ARV7

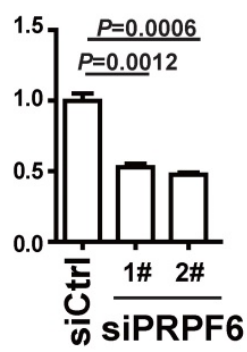

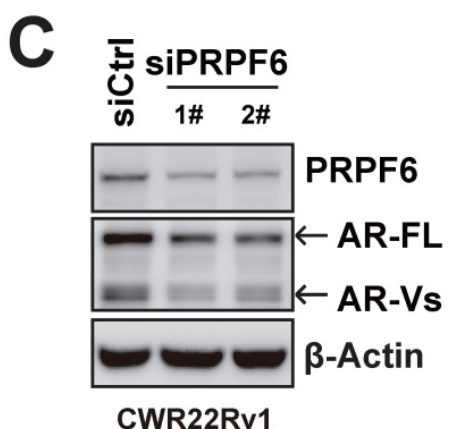

E
D
E LNCaP

$\begin{array}{llll}0 & 1 & 2 & 4(\mu \mathrm{g}) \text { Myc-PFPF6 }\end{array}$
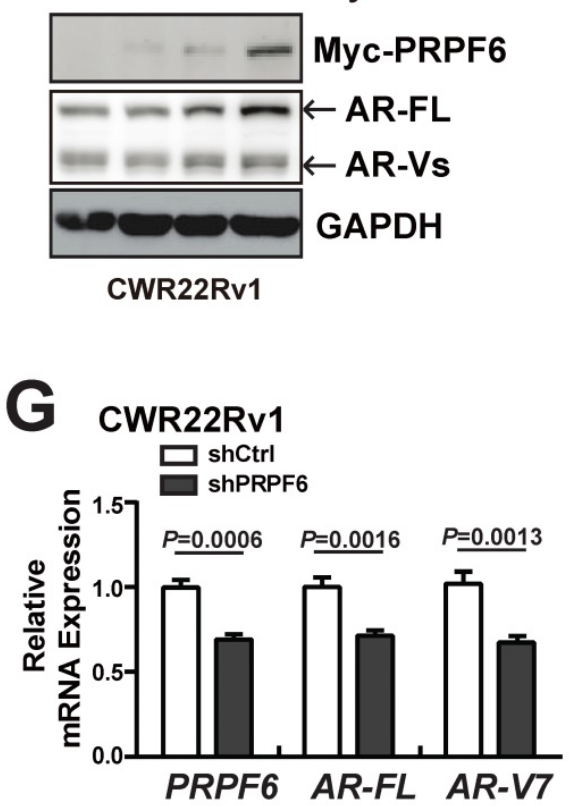

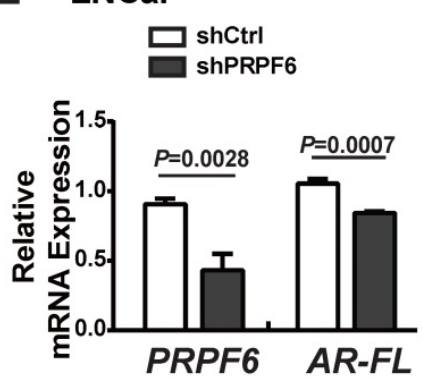

H

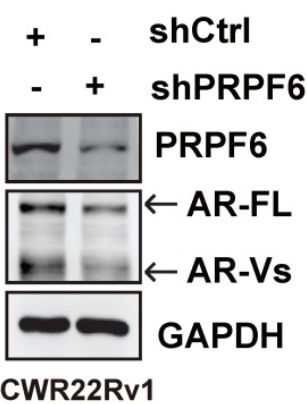

LNCaP
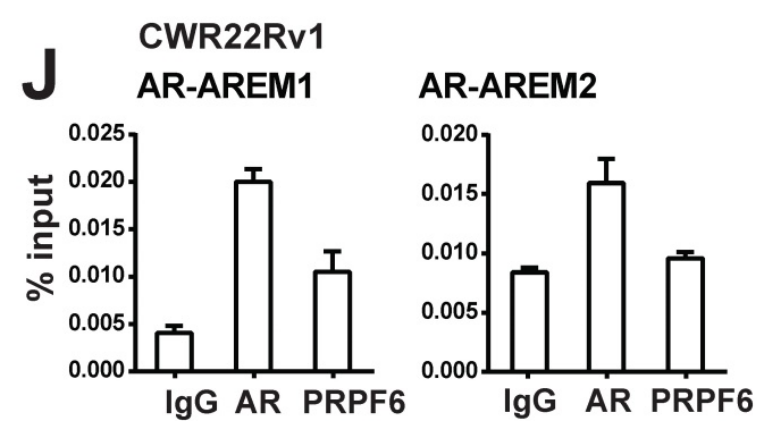

Figure 5. PRPF6 promotes the expressions of AR-FL and AR-V7. A and B. Knockdown of PRPF6 downregulated mRNA expressions of AR-FL in LNCaP cells (A), and downregulated mRNA expression of AR-FL and AR-V7 in CWR22Rv1 cells (B). Cells were transfected with two independent siRNAs against PRPF6 (siPRPF6) or control siRNA (siCtrl). After $48 \mathrm{hrs}$, cells were collected for RNA extraction and real-time PCR were performed. Student's t-test was performed. C. Knockdown of PRPF6 downregulated protein expressions of AR-FL and AR-Vs in CWR22Rvl cells. CWR22Rvl cells were transfected with siCtrl or siPRPF6. After 48 hrs, cells were collected for protein extraction and subjected to western blot analysis using antibodies against PRPF6 (Santa Cruz) and AR (Proteintech). $\beta$-Actin was used as internal control. D. Ectopic expression of PRPF6 upregulated protein expressions of AR-FL and AR-Vs in CWR22Rv1 cells. CWR22Rv1 cells were transfected with different amounts of Myc-tagged PRPF6 expression plasmids or control vector. After $48 \mathrm{hrs}$, cells were collected for protein extraction and subjected to western blot analysis using anti-AR (Proteintech) and the indicated antibodies. GAPDH was used as internal control. $\mathbf{E}$ and F. Impacts of lentivirus-mediated PRPF6 knockdown on the expression of AR-FL in LNCaP cells. LNCaP cells with shPRPF6 or shCtrl were collected for RNA extraction and real-time PCR were performed (E), or protein extraction and subjected to western blot analysis using antibodies against PRPF6 (Santa Cruz) and AR (Proteintech) (F). G. Impacts of lentivirus-mediated PRPF6 knockdown on the mRNA expressions of AR-FL and AR-V7 in CWR22Rv1 cell. CWR22Rv1 cell with shPRPF6 or shCtrl were collected for RNA extraction and subjected to real-time PCR. $\mathbf{H}$. Impacts of lentivirus-mediated PRPF6 knockdown on the protein expressions of AR-FL and AR-Vs in CWR22Rvl cells. Cells were infected with shPRPF6 or shCtrl, and then were collected for protein extraction and subjected to western blot analysis using antibodies against PRPF6 (Santa Cruz) and AR (Proteintech). GAPDH was used as internal control. I. Schematic represents AR binding cis-elements in AR gene. J. PRPF6 was recruited to AR-binding cis-elements in AR gene. CWR22Rvl cells were subjected to ChIP assays using normal lgG, anti-AR (Invitrogen) or anti-PRPF6 (Bethyl). 
A

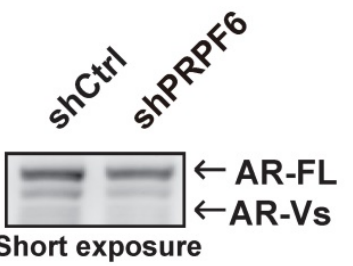

AR-Vs

Long exposure

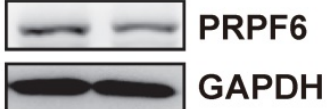

LNCaP-Al

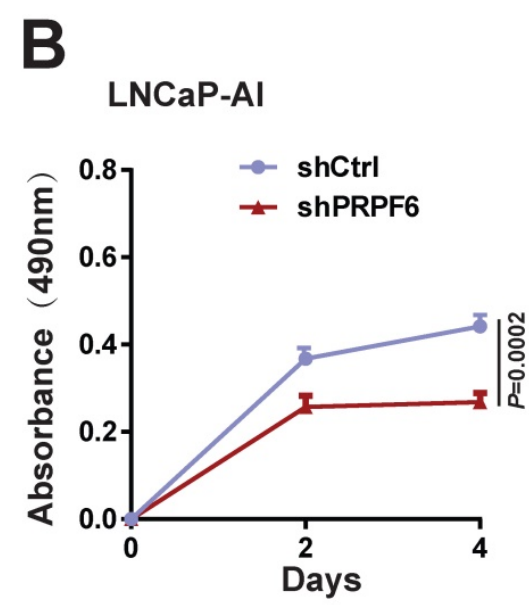

C
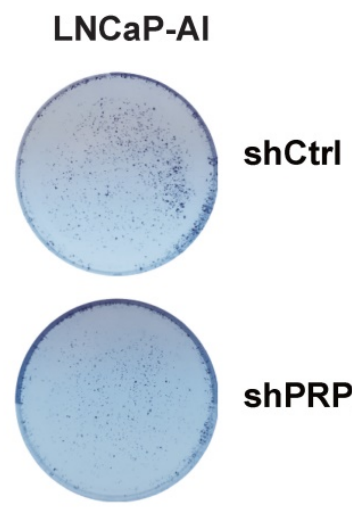

shPRPF6
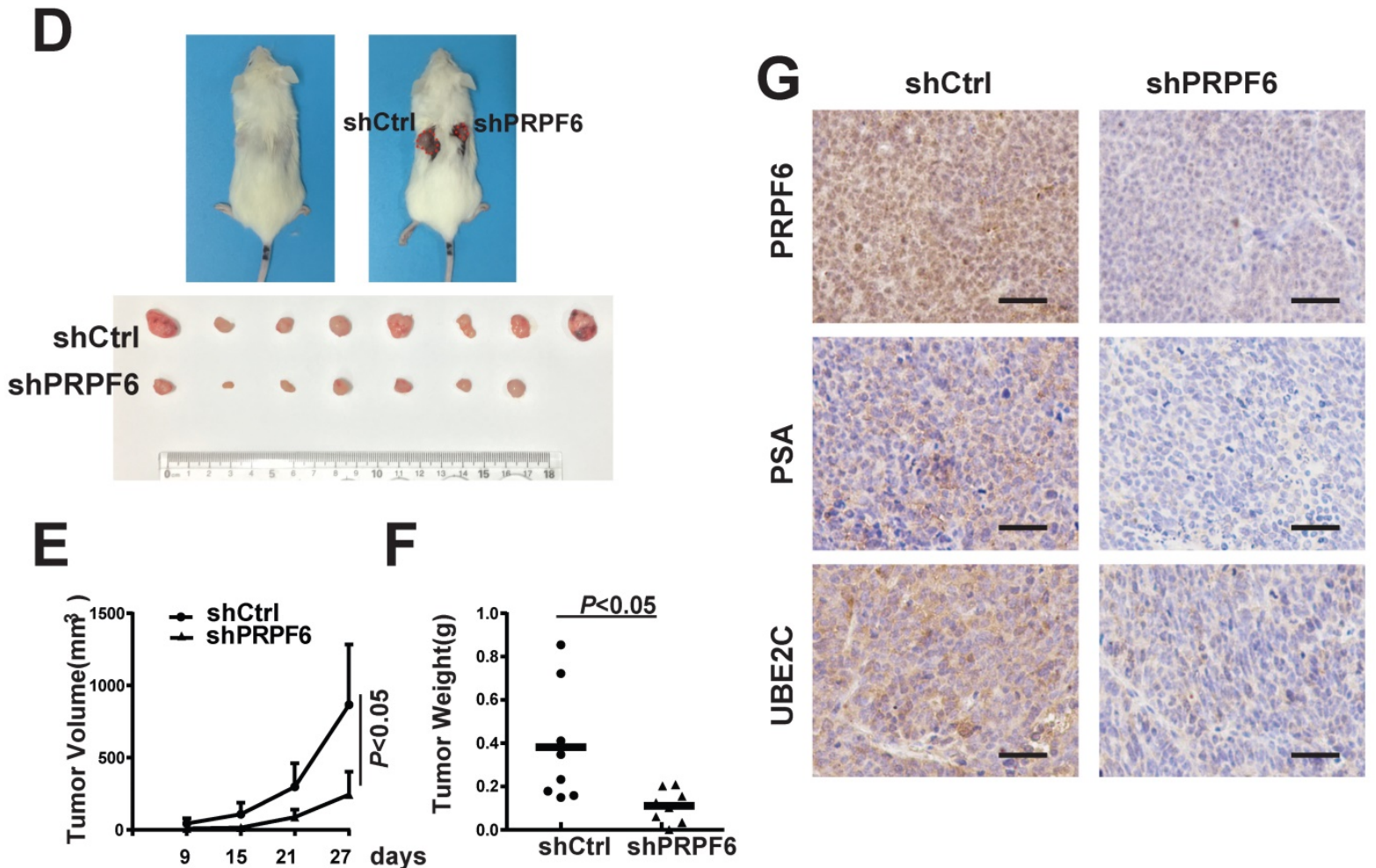

Figure 6. PRPF6 promotes cell growth under androgen-depleted condition. A. Impacts of lentivirus-mediated PRPF6 knockdown on the protein expressions in LNCaP-Al cells. LNCaP-Al cells were infected with shPRPF6 or shCtrl, and then were collected for protein extraction and subjected to western blot analysis using antibodies using anti-PRPF6 (Proteintech), anti-AR (Proteintech) and GAPDH. B. PRPF6 knockdown repressed cell proliferation of LNCaP-AI cells. LNCaP-AI cells (2500 per well) with shPRPF6 or shCtrl were cultured in medium with $5 \%$ CSS. After indicated time, MTS reagent was added, then absorbance at $490 \mathrm{~nm}$ was measured and plotted. Data were means \pm SD of three independent experiments. Student's $t$-test was performed. C. PRPF6 knockdown inhibited cell growth of LNCaP-AI cells under androgen-depleted condition. LNCaP-Al cells (5000 pre well) with shPRPF6 or shCtrl were infected with shPRPF6 or shCtrl, and then were cultured for 14 days. D. PRPF6 knockdown inhibited cell growth in xenograft mice under castration condition. Castration operation was performed on 5 -week-old male NOD/SCID mice. After one week, CWR22Rvl cells with shPRPF6 or shCtrl were injected subcutaneously into castrated male mice. Representative photograph of mice with tumor xenografts (upper panel). Photograph of all xenograft tumors derived from CWR22Rv1 cells with shPRPF6 or shCtrl (lower panel). E. Tumor volumes of xenografts in castrated male mice were shown. The volume of xenograft tumors derived from CWR22Rvl cells with shPRPF6 or shCtrl were measured every 6 days. Data represent means \pm SD. Student's $t$-test was performed. F. Final tumor weights of xenograft tumors derived from CWR22Rvl cells with shPRPF6 or shCtrl in castrated male mice were measured and shown. Horizontal lines represent the mean values. Student's $t$-test was performed. G. Expressions of indicated proteins in xenograft tumors were detected by IHC assays. Scale bars, $100 \mu \mathrm{m}$.

\section{PRPF6 is highly expressed in clinical prostate cancer samples}

Having revealed the involvement of PRPF6 in AR signaling and growth of prostate cancer cells, we next examined the expression of PRPF6 in clinical prostate cancer samples. In good agreement with upregulation of AR-FL and AR-Vs expression by PRPF6 (Figure 5), we found that mRNA expression of PRPF6 was positively correlated with that of $A R$ in prostate cancer patients of TCGA database using the Gene Expression Profiling Interactive Analysis (GEPIA) [36], suggesting PRPF6 participate in regulation of $A R$ transcription in clinical prostate 
cancer tissues (Figure 7A). We then examined the expression of PRPF6 in 71 pairs of prostate cancer tissues and the matched adjacent noncancerous tissues by immunohistochemical stain (IHC). The results showed that PRPF6 primarily localized in nucleus, as well as mild cytoplasmic staining was also observed (Figure 7B). The stain intensity of PRPF6 was increased in prostate cancer tissues compared with the matched adjacent noncancerous tissues (Figure 7C). Further, in the above 71 prostate cancer tissues and another 20 prostate cancer tissues, PRPF6 expression was higher in prostate cancers with higher Gleason scores (Figure 7D). These results suggested that PRPF6 might play a role in promotion of clinical prostate cancers.
A

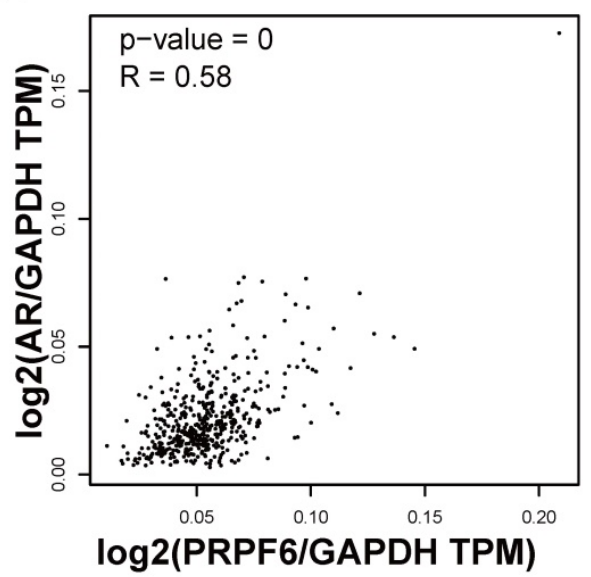

0

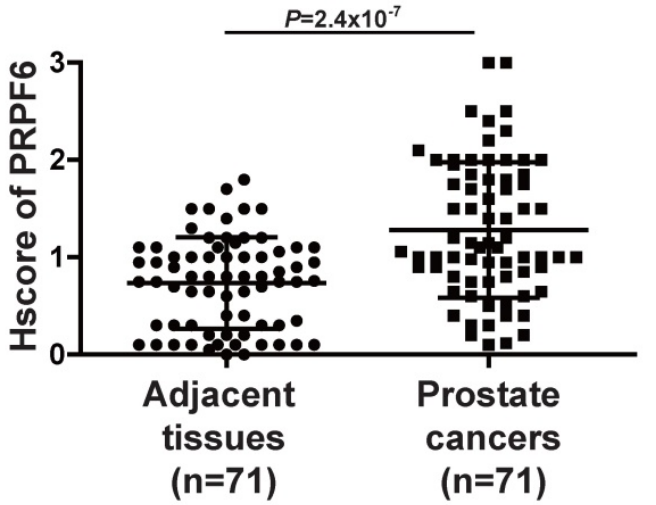

D

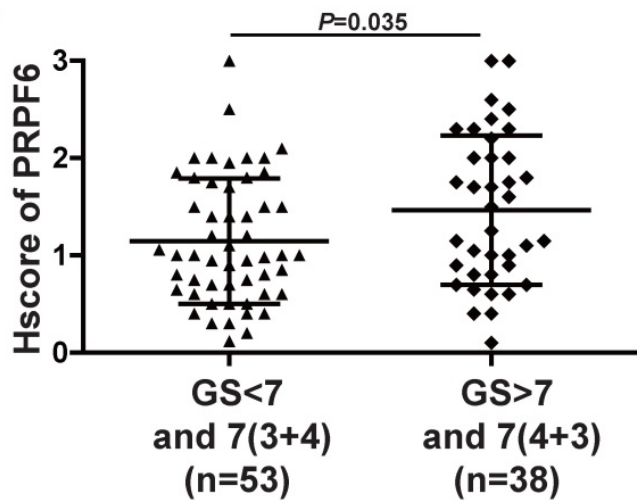

B

GS7(3+4)

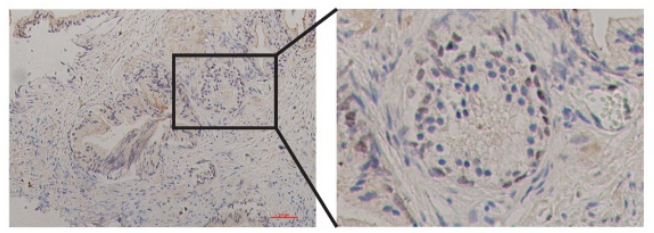

BPH

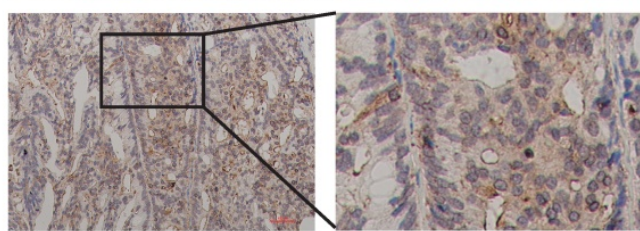

GS9(5+4)

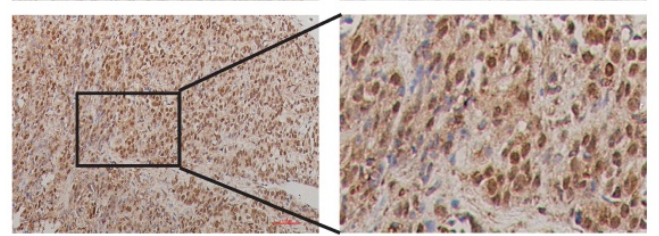

E
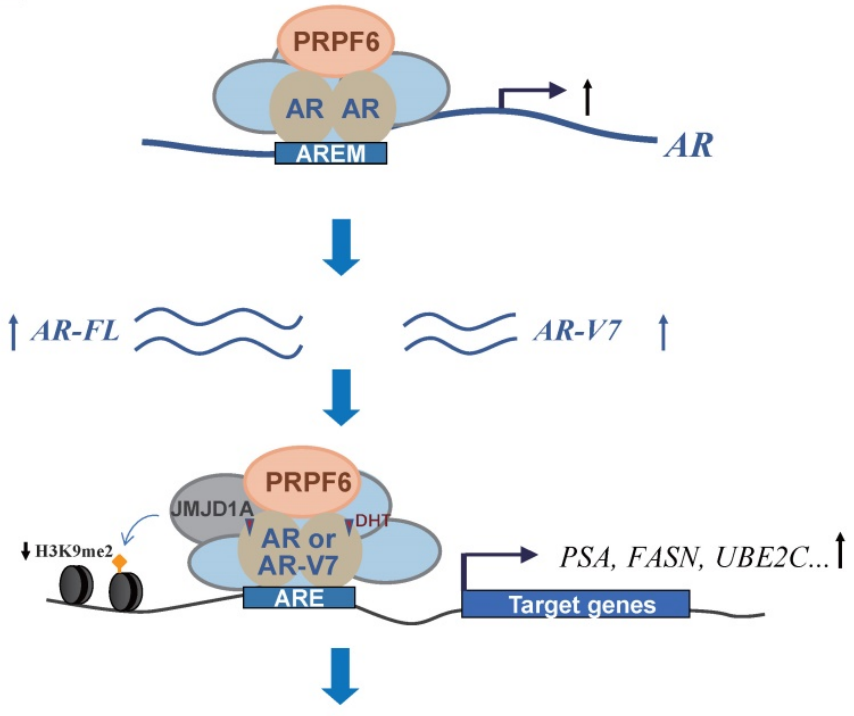

Promote PCa/CRPC progression

Figure 7. PRPF6 expressions in human prostate cancers and adjacent noncancerous tissues. A. Pearson correlation between mRNA expression of PRPF6 and that of $A R$ in prostate cancer from TCGA database. The relative level of PRPF6 normalized by GAPDH was plotted against that of AR via GEPIA (http://gepia.cancer-pku.cn/). B. Representative images of PRPF6 immunohistochemical staining in human prostate cancers and adjacent noncancerous tissues. BPH, benign prostate hyperplasia. GS, Gleason score. Scale bars, $100 \mu \mathrm{m}$. C. Increased expression of PRPF6 in clinical prostate cancer tissues, compared with the matched adjacent non-cancerous tissues. Horizontal lines represent the means \pm SD. Student's $t$-test was performed. D. PRPF6 expression in prostate cancer samples with different Gleason score. Horizontal lines represent the means \pm SD. Student's $t$-test was performed. E. Schematic representation of the functions of PRPF6 in prostate cancer and CRPC. 


\section{Discussion}

It has been demonstrated that several splicing factors are upregulated and drives the progression of prostate cancer $[5,6]$. In this study, we found that PRPF6 is recruited to cis-response elements on AR target genes, thereby modulating histone modification level and enhancing AR-induced transcriptional activity. On the other hand, PRPF6 promotes the expression of AR-FL and AR-V7 (Figure 7E). Our data demonstrated that PRPF6 plays a vital role in modulation of oncogenic AR signaling pathway and promotes the progression of prostate cancer and CRPC.

JMJD1A functions as a key coactivator for AR by epigenetic regulation of $\mathrm{H} 3 \mathrm{~K} 9$ methylation marks [31]. Knockdown of PRPF6 increased histone H3K9me2 level at ARE region (Figure 4A-4C), and weakened the interaction between JMJD1A and AR (Figure 4E and 4F). Together with a stronger PRPF6-JMJD1A interaction could be detected under DHT treatment (Figure 4D), our data indicates that PRPF6 could facilitate the association of JMJD1A with AR, thereby promoting AR-mediated transcription. It is reported that PRPF6 has been known to function as a component in the protein complexes NCoR2 complex with histone deacetylase activity [27]. Here, our results indicated that PRPF6, within a complex that contains JMJD1A, may contribute to AR-mediated transactivation via modulation of histone H3K9 methylation. It has also been reported that brahma related gene 1 (BRG1) associates JMJD1A as well as SET1A, a histone H3K4 methyltransferase, plays a critical role in trans-activation of CSF1 [37]. In addition to histone $\mathrm{H} 3 \mathrm{~K} 9 \mathrm{me}$, our results showed PRPF6 knockdown resulted in a reduction in histone H3K4me3 levels at ARE region (Figure 4A-4C), implying that histone H3K4 methyltransferase may be associated with PRPF6-JMJD1A-AR complex, on the other hand, there might also be a crosstalk between demethylation of H3K9 and methylation of H3K4.

In line with the previous report that transactivation domain of PRPF6 exists within 78-495 aa residues [25], our results showed that PRPF6 full length (FL), PRPF6 5TPR (1-482 aa) and PRPF6 10TPR (1-640 aa) showed similar ability to enhance AR-FLmediated transactivation as PRPF6 FL (Figure 3D).It is notable that truncated mutant PRPF6 15TPR (1-809 aa), which exhibited obvious speckle localization (Figure 2E), significantly enhanced AR-FL-mediated transactivation at a comparable expression level to PRPF6 FL (Figure 3D and Supplementary Figure S4). Subnuclear speckles are discovered as sites for splicing factor storage and modification [38]. Increasing evidences demonstrate that, in many cases, there is a reciprocal relationship between splicing and transcription [39, 40]. Studies are showing that splicing can affect chromatin organization and histone modification [41, 42]. Although, relying on our data, we were unable to verify whether the increased function in transcriptional regulation of PRPF6 15TPR is related to splicing process, which remains to be elucidated in detailed future studies. Besides a cofactor of AR-FL, our data showed PRPF6 also co-activates AR-V7 mediated transcription (Figure 3), indicating that PRPF6 plays an important role in enhancing AR-FL and AR-V7 action in prostate cancer and CRPC.

AR-V7 generation could be facilitated by aberrant splicing and/or $A R$ genomic structural rearrangement $[3,4,18,19]$. In addition, it has been considered that alternative splicing process of $A R-V 7$ couples with transcription of $A R$ gene [4, 19]. In line with this, levels of $A R-F L$ and $A R-V 7$ transcripts are often tightly correlated in individual clinical specimens and xenograft models [16, 43, 44]. Several splicing factors have been proposed as regulators of $A R-V 7$ splicing. U2AF65 and SRSF1 act as 'pioneer' factors, directing the recruitment of the spliceosome and increasing the expression of $A R-V 7$ [19]. Histone lysine demethylase $4 \mathrm{~B}$ (KDM4B) is phosphorylated by PKA in response to androgen deprivation, which in turn elicits its binding to U2 snRNP SF3B3, promoting $A R-V 7$ expression by regulating the alternative splicing of $A R$ [45]. PRPF6 has been demonstrated to alter the constitutive and alternative splicing of a discrete number of genes in colon cancer [24]. In this study, we provided the evidence that PRPF6 also binds cis-regulatory elements on $A R$ gene to enhancing the transcription of $A R$ gene itself, thereby promoting the expression of AR-FL or AR-V7 (Figure 5). Our findings are also in good agreement with the previously proposed idea that activated steroid receptors bind to target DNA response elements and promote the recruitment of coregulators that are involved in both transcription and splicing regulation, thereby mediating effects on transcription and splice processing [46-49].

In summary, our results have demonstrated that PRPF6 regulates oncogenic AR signaling pathway in prostate cancer-derived cell lines, PRPF6 depletion reduces tumor growth of prostate cancer-derived cell lines even under the castration condition in mice, and PRPF6 is highly expressed in clinical prostate cancer samples. Our data support a previously unknown role of PRPF6 during prostate cancer progression, which may provide an insight to potential therapeutic strategies for prostate cancer, especially for CRPC. 


\section{Abbreviations}

AR: androgen receptor; PCa: prostate cancer; AR-V7: AR-variant 7; CRPC: castration-resistant prostate cancer; ADT: androgen depletion therapy; mRNA: messenger RNA; pre-mRNA: precursor messenger RNA; snRNP: small nuclear ribonucleoprotein; PRPF6: pre-mRNA processing factor 6; CSS: charcoal/dextran-stripped serum; RNAi: RNA interference; RIP: RNA immunoprecipitation; ChIP: chromatin immunoprecipitation; IHC: immunohistochemistry; SS: splice site; DHT: dihydrotestosterone; TPR: tetratricopeptide repeat; PSA: prostate-specific antigen; KLK2: kallikrein related peptidase 2; FASN: fatty acid synthase; TMPRSS2: transmembrane protease serine 2; UBE2C: ubiquitin-conjugating enzyme E2 C; BMPRIB: bone morphogenetic protein type IB receptor; ELOVL5: ELOVL fatty acid elongase 5; HPGD: 15-hydroxyprostaglandin dehydrogenase; SLC45A3: solute carrier family 45 a3 member; ACPP: acid phosphatase 3; ARE: AR-responsive element; SRSF: serine-arginine rich splicing factor; HNRNP: heterogeneous nuclear ribonucleoprotein; PSF/SFPQ: splicing factor proline and glutamine-rich; KDM4B: histone lysine demethylase 4B; JMJD1A: histone demethylase jumonji domain containing 1A; ASC: activating signal cointegrator; RNAPII: RNA polymerase II.

\section{Supplementary Material}

Supplementary figures and tables.

http://www.ijbs.com/v17p0188s1.pdf

\section{Acknowledgements}

This work was supported by grants from National Natural Science Foundation of China (31871286 for Yue Zhao, 81872015 for Chunyu Wang, 31701102 for Shengli Wang, 81702800 for Renlong Zou, 81902889 for Wensu Liu); Foundation for Special Professor of Liaoning Province (the $5^{\text {th }}$ batch) for Yue Zhao; Natural Science Foundation of Liaoning Province (20180530072) for Chunyu Wang.

\section{Author contributions}

Wei Liu, Chunyu Wang and Shengli Wang performed experiments, analyzed the data and wrote the manuscript; Shan Yu, Ning Sun, Ge Sun, Manlin Wang, Renlong Zou Wensu Liu, Lin Lin and Huijuan Song performed experiments; Kai Zeng and Zining Jin conducted statistical analyses; Yue Zhao conceived the project, wrote and revised manuscript. All authors read the approved the final manuscript.

\section{Competing Interests}

The authors have declared that no competing interest exists.

\section{References}

1. Ferlay J, Colombet M, Soerjomataram I, Mathers C, Parkin DM, Pineros M, et al. Estimating the global cancer incidence and mortality in 2018: GLOBOCAN sources and methods. Int J Cancer. 2019; 144: 1941-53.

2. Attard G, Parker C, Eeles RA, Schroder F, Tomlins SA, Tannock I, et al. Prostate cancer. Lancet. 2016; 387: 70-82.

3. Hu R, Dunn TA, Wei S, Isharwal S, Veltri RW, Humphreys E, et al. Ligand-independent androgen receptor variants derived from splicing of cryptic exons signify hormone-refractory prostate cancer. Cancer Res. 2009; 69: $16-22$.

4. Li Y, Alsagabi M, Fan D, Bova GS, Tewfik AH, Dehm SM. Intragenic rearrangement and altered RNA splicing of the androgen receptor in a cell-based model of prostate cancer progression. Cancer Res. 2011; 71: 2108-17.

5. Paschalis A, Sharp A, Welti JC, Neeb A, Raj GV, Luo J, et al. Alternative splicing in prostate cancer. Nat Rev Clin Oncol. 2018; 15: 663-75.

6. Takayama KI. Splicing Factors Have an Essential Role in Prostate Cancer Progression and Androgen Receptor Signaling. Biomolecules. 2019; 9.

7. Robinson D, Van Allen EM, Wu YM, Schultz N, Lonigro RJ, Mosquera JM, et al. Integrative clinical genomics of advanced prostate cancer. Cell. 2015; 161: 1215-28.

8. Cato L, Neeb A, Sharp A, Buzon V, Ficarro SB, Yang L, et al. Development of Bag- $1 \mathrm{~L}$ as a therapeutic target in androgen receptor-dependent prostate cancer. Elife. 2017; 6

9. Rodriguez-Bravo V, Pippa R, Song WM, Carceles-Cordon M, Dominguez-Andres A, Fujiwara N, et al. Nuclear Pores Promote Lethal Prostate Cancer by Increasing POM121-Driven E2F1, MYC, and AR Nuclear Import. Cell. 2018; 174: 1200-15 e20.

10. Takeda DY, Spisak S, Seo JH, Bell C, O'Connor E, Korthauer K, et al. A Somatically Acquired Enhancer of the Androgen Receptor Is a Noncoding Driver in Advanced Prostate Cancer. Cell. 2018; 174: 422-32 e13.

11. Culig Z. Androgen Receptor Coactivators in Regulation of Growth and Differentiation in Prostate Cancer. J Cell Physiol. 2016; 231: 270-4.

12. Wang C, Sun H, Zou R, Zhou T, Wang S, Sun S, et al. MDC1 functionally identified as an androgen receptor co-activator participates in suppression of prostate cancer. Nucleic Acids Res. 2015; 43: 4893-908.

13. Sun S, Zhong X, Wang C, Sun H, Wang S, Zhou T, et al. BAP18 coactivates androgen receptor action and promotes prostate cancer progression. Nucleic Acids Res. 2016; 44: 8112-28.

14. Moon SJ, Jeong BC, Kim HJ, Lim JE, Kwon GY, Kim JH. DBC1 promotes castration-resistant prostate cancer by positively regulating DNA binding and stability of AR-V7. Oncogene. 2018; 37: 1326-39.

15. Qu Y, Dai B, Ye D, Kong Y, Chang K, Jia Z, et al. Constitutively active AR-V7 plays an essential role in the development and progression of castration-resistant prostate cancer. Sci Rep. 2015; 5: 7654.

16. Sharp A, Coleman I, Yuan W, Sprenger C, Dolling D, Rodrigues DN, et al. Androgen receptor splice variant-7 expression emerges with castration resistance in prostate cancer. J Clin Invest. 2019; 129: 192-208.

17. Antonarakis ES, Lu C, Wang H, Luber B, Nakazawa M, Roeser JC, et al. AR-V7 and resistance to enzalutamide and abiraterone in prostate cancer. $\mathrm{N}$ Engl J Med. 2014; 371: 1028-38.

18. Henzler C, Li Y, Yang R, McBride T, Ho Y, Sprenger C, et al. Truncation and constitutive activation of the androgen receptor by diverse genomic rearrangements in prostate cancer. Nat Commun. 2016; 7: 13668.

19. Liu LL, Xie N, Sun S, Plymate S, Mostaghel E, Dong X. Mechanisms of the androgen receptor splicing in prostate cancer cells. Oncogene. 2014; 33: 3140-50.

20. Dvinge H, Kim E, Abdel-Wahab O, Bradley RK. RNA splicing factors as oncoproteins and tumour suppressors. Nat Rev Cancer. 2016; 16: 413-30.

21. Climente-Gonzalez H, Porta-Pardo E, Godzik A, Eyras E. The Functional Impact of Alternative Splicing in Cancer. Cell Rep. 2017; 20: 2215-26.

22. Seiler M, Peng S, Agrawal AA, Palacino J, Teng T, Zhu P, et al. Somatic Mutational Landscape of Splicing Factor Genes and Their Functional Consequences across 33 Cancer Types. Cell Rep. 2018; 23: 282-96 e4.

23. Liu S, Rauhut R, Vornlocher HP, Luhrmann R. The network of protein-protein interactions within the human U4/U6.U5 tri-snRNP. RNA. 2006; 12: 1418-30.

24. Adler AS, McCleland ML, Yee S, Yaylaoglu M, Hussain S, Cosino E, et al. An integrative analysis of colon cancer identifies an essential function for PRPF6 in tumor growth. Genes Dev. 2014; 28: 1068-84.

25. Zhao $\mathrm{Y}$, Goto $\mathrm{K}$, Saitoh $\mathrm{M}$, Yanase $\mathrm{T}$, Nomura $\mathrm{M}$, Okabe $\mathrm{T}$, et al Activation function-1 domain of androgen receptor contributes to the interaction between subnuclear splicing factor compartment and nuclear receptor compartment. Identification of the 102 U5 small nuclear ribonucleoprotein particle-binding protein as a coactivator for the receptor. J Biol Chem. 2002; 277: 30031-9.

26. Wang C, Li Y, Zhang H, Liu F, Cheng Z, Wang D, et al. Oncogenic PAK4 regulates Smad2/3 axis involving gastric tumorigenesis. Oncogene. 2014; 33: 3473-84.

27. Dellaire G, Makarov EM, Cowger JJ, Longman D, Sutherland HG, Luhrmann $\mathrm{R}$, et al. Mammalian PRP4 kinase copurifies and interacts with components of both the U5 snRNP and the N-CoR deacetylase complexes. Mol Cell Biol. 2002; 22: 5141-56. 
28. de Almeida SF, Grosso AR, Koch F, Fenouil R, Carvalho S, Andrade J, et al. Splicing enhances recruitment of methyltransferase HYPB/Setd2 and methylation of histone H3 Lys36. Nat Struct Mol Biol. 2011; 18: 977-83.

29. Kim S, Kim H, Fong N, Erickson B, Bentley DL. Pre-mRNA splicing is a determinant of histone H3K36 methylation. Proc Natl Acad Sci U S A. 2011; 108: 13564-9.

30. Yamane K, Toumazou C, Tsukada Y, Erdjument-Bromage H, Tempst P, Wong $\mathrm{J}$, et al. JHDM2A, a JmjC-containing H3K9 demethylase, facilitates transcription activation by androgen receptor. Cell. 2006; 125: 483-95.

31. Tateishi K, Okada Y, Kallin EM, Zhang Y. Role of Jhdm2a in regulating metabolic gene expression and obesity resistance. Nature. 2009; 458: 757-61.

32. Fan L, Zhang F, Xu S, Cui X, Hussain A, Fazli L, et al. Histone demethylase JMJD1A promotes alternative splicing of AR variant 7 (AR-V7) in prostate cancer cells. Proc Natl Acad Sci U S A. 2018; 115: E4584-E93.

33. Mahajan K, Malla P, Lawrence HR, Chen Z, Kumar-Sinha C, Malik R, et al. ACK1/TNK2 Regulates Histone H4 Tyr88-phosphorylation and AR Gene Expression in Castration-Resistant Prostate Cancer. Cancer Cell. 2017; 31: $790-803$ e8.

34. Cato L, de Tribolet-Hardy J, Lee I, Rottenberg JT, Coleman I, Melchers D, et al. ARv7 Represses Tumor-Suppressor Genes in Castration-Resistant Prostate Cancer. Cancer Cell. 2019; 35: 401-13 e6.

35. Wang Y, Wang Y, Liu Q, Xu G, Mao F, Qin T, et al. Comparative RNA-seq analysis reveals potential mechanisms mediating the conversion to androgen independence in an LNCaP progression cell model. Cancer Lett. 2014; 342: $130-8$.

36. Tang Z, Li C, Kang B, Gao G, Li C, Zhang Z. GEPIA: a web server for cancer and normal gene expression profiling and interactive analyses. Nucleic Acids Res. 2017; 45: W98-W102.

37. Shao J, Weng X, Zhuo L, Yu L, Li Z, Shen K, et al. Angiotensin II induced CSF1 transcription is mediated by a crosstalk between different epigenetic factors in vascular endothelial cells. Biochim Biophys Acta Gene Regul Mech. 2019; 1862: 1-11.

38. Galganski L, Urbanek MO, Krzyzosiak WJ. Nuclear speckles: molecular organization, biological function and role in disease. Nucleic Acids Res. 2017; 45: 10350-68.

39. Chathoth KT, Barrass JD, Webb S, Beggs JD. A splicing-dependent transcriptional checkpoint associated with prespliceosome formation. Mol Cell. 2014; 53: 779-90.

40. de Almeida SF, Carmo-Fonseca M. Reciprocal regulatory links between cotranscriptional splicing and chromatin. Semin Cell Dev Biol. 2014; 32: 2-10.

41. Keren-Shaul H, Lev-Maor G, Ast G. Pre-mRNA splicing is a determinant of nucleosome organization. PLoS One. 2013; 8: e53506.

42. Bieberstein NI, Carrillo Oesterreich F, Straube K, Neugebauer KM. First exon length controls active chromatin signatures and transcription. Cell Rep. 2012; 2: $62-8$.

43. Watson PA, Chen YF, Balbas MD, Wongvipat J, Socci ND, Viale A, et al. Constitutively active androgen receptor splice variants expressed in castration-resistant prostate cancer require full-length androgen receptor. Proc Natl Acad Sci U S A. 2010; 107: 16759-65.

44. Yu Z, Chen S, Sowalsky AG, Voznesensky OS, Mostaghel EA, Nelson PS, et al. Rapid induction of androgen receptor splice variants by androgen deprivation in prostate cancer. Clin Cancer Res. 2014; 20: 1590-600.

45. Duan L, Chen Z, Lu J, Liang Y, Wang M, Roggero CM, et al. Histone lysine demethylase KDM4B regulates the alternative splicing of the androgen receptor in response to androgen deprivation. Nucleic Acids Res. 2019; 47: 11623-36.

46. Auboeuf D, Honig A, Berget SM, O'Malley BW. Coordinate regulation of transcription and splicing by steroid receptor coregulators. Science. 2002; 298 : 416-9.

47. Monsalve M, Wu Z, Adelmant G, Puigserver P, Fan M, Spiegelman BM. Direct coupling of transcription and mRNA processing through the thermogenic coactivator PGC-1. Mol Cell. 2000; 6: 307-16.

48. Auboeuf D, Dowhan DH, Li X, Larkin K, Ko L, Berget SM, et al. CoAA, a nuclear receptor coactivator protein at the interface of transcriptional coactivation and RNA splicing. Mol Cell Biol. 2004; 24: 442-53.

49. Dowhan DH, Hong EP, Auboeuf D, Dennis AP, Wilson MM, Berget SM, et al. Steroid hormone receptor coactivation and alternative RNA splicing by U2AF65-related proteins CAPERalpha and CAPERbeta. Mol Cell. 2005; 17: 429-39. 